\title{
Nickel-Catalyzed Reductive Cross-Coupling of Aryl Halides with Alkyl Halides
}

Daniel A. Everson, Ruja Shrestha, and Daniel J. Weix*.

Department of Chemistry, University of Rochester, Rochester, NY 14627

Supporting Information 
Table of Contents

I. General S3

II. Optimization and Control Reactions $\quad$ S5

$\begin{array}{ll}\text { III. Preparation of Organohalides } & \text { S8 }\end{array}$

IV. Reductive Cross-Coupling Reactions $\quad$ S8

V. Spectra

4-iodo(phenyl)methanol ${ }^{1} \mathrm{H}$ NMR $\quad \mathrm{S} 16$

4-iodo(phenyl)methanol ${ }^{13} \mathrm{C}$ NMR $\quad \mathrm{S} 17$

Compound 3a ${ }^{1} \mathrm{H}$ NMR $\quad \mathrm{S} 18$

\begin{tabular}{ll} 
Compound 3a ${ }^{13} \mathrm{C}$ NMR & $\mathrm{S} 19$ \\
\hline
\end{tabular}

Compound $\mathbf{3 b}{ }^{1} \mathrm{H}$ NMR $\quad \mathrm{S} 20$

Compound $\mathbf{3 b}{ }^{13} \mathrm{C}$ NMR $\quad$ S21

Compound $3 \mathbf{c}^{1} \mathrm{H}$ NMR $\quad$ S22

Compound 3c ${ }^{13} \mathrm{C}$ NMR $\quad$ S23

Compound 3c ${ }^{19}$ F NMR $\quad$ S24

Compound 3d ${ }^{1} \mathrm{H}$ NMR $\quad$ S25

Compound 3d ${ }^{13} \mathrm{C}$ NMR $\quad$ S26

Compound 3e ${ }^{1} \mathrm{H}$ NMR $\quad$ S27

Compound $3 e^{13} \mathrm{C}$ NMR $\quad$ S28

$\begin{array}{ll}\text { Compound } \mathbf{3 f}{ }^{1} \mathrm{H} \text { NMR } & \text { S29 }\end{array}$

Compound $3 f^{13} \mathrm{C}$ NMR $\quad$ S30

Compound $\mathbf{3 g}{ }^{1} \mathrm{H}$ NMR $\quad$ S31

Compound 3g ${ }^{13} \mathrm{C}$ NMR $\quad$ S32

Compound $\mathbf{3 h}{ }^{1} \mathrm{H}$ NMR $\quad$ S33

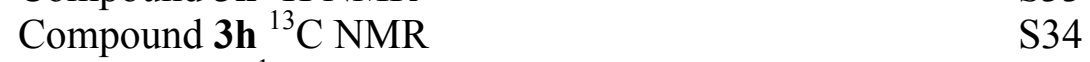

Compound 3i ${ }^{1} \mathrm{H}$ NMR $\quad \mathrm{S} 35$

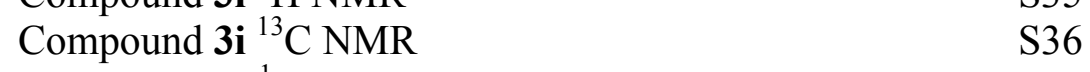

Compound $\mathbf{3 j}{ }^{1} \mathrm{H}$ NMR $\quad$ S37

$\begin{array}{ll}\text { Compound } \mathbf{3 j}{ }^{13} \mathrm{C} \text { NMR } & \mathrm{S} 38\end{array}$

Compound $\mathbf{3 k}{ }^{1} \mathrm{H}$ NMR $\quad$ S39

Compound 3k ${ }^{13} \mathrm{C}$ NMR $\quad \mathrm{S} 40$

Compound $31{ }^{1} \mathrm{H}$ NMR $\quad \mathrm{S} 41$

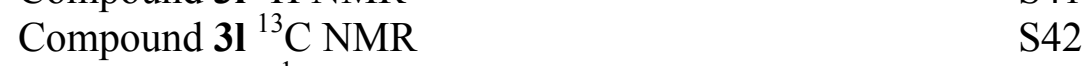

Compound $\mathbf{3 m}{ }^{1} \mathrm{H}$ NMR $\quad \mathrm{S} 43$

Compound 3m ${ }^{13} \mathrm{C}$ NMR $\quad \mathrm{S} 44$

$\begin{array}{ll}\text { Compound 3n }{ }^{1} \mathrm{H} \text { NMR } & \mathrm{S} 45\end{array}$

Compound 3n ${ }^{13} \mathrm{C} \mathrm{NMR} \quad \mathrm{S} 46$

$\begin{array}{ll}\text { Compound 3o }{ }^{1} \mathrm{H} \text { NMR } & \mathrm{S} 47\end{array}$

Compound 3o ${ }^{13} \mathrm{C}$ NMR $\quad \mathrm{S} 48$

Compound 3p ${ }^{1} \mathrm{H}$ NMR $\quad$ S49

$\begin{array}{ll}\text { Compound 3p }{ }^{13} \mathrm{C} \text { NMR } & \text { S50 }\end{array}$ 


\section{General}

\section{Chemicals.}

$\mathrm{NiI}_{2} \times \mathrm{xH}_{2} \mathrm{O} \quad(\mathrm{x}=3.5$ by elemental analysis $), \quad \mathrm{Ni}(\operatorname{cod})_{2}$ and 1,2bis(diphenylphosphino)benzene were purchased from Strem Chemicals and used as received. $\mathrm{NiCl}_{2}$ (dme) was either purchased from Strem or made according to the literature procedure. ${ }^{1}$ It is important to note that the stoichiometry of both $\mathrm{NiI}_{2}$ hydrate and $\mathrm{NiCl}_{2}(\mathrm{dme})$ are variable (our $\mathrm{NiCl}_{2}(\mathrm{dme})$ varied from 0.1 to $0.7 \mathrm{dme}$ and the dme is easily lost upon exposure to vacuum). We found that excess nickel is not detrimental to the isolated yield of product, but we obtained lower yields in reactions employing excess ligands. Therefore, we employed slight excess $(0.107$ equiv [Ni] to $0.100 \mathrm{~mol} \%$ [ligands]) in our reactions to account for the variable nickel stoichiometry.

4,4'-Di-tert-butyl-2,2'-dipyridyl, manganese powder (325 mesh), anhydrous pyridine, dichloromethane, DMF, pinacol, di-tert-butyl dicarbonate, benzyl chloroformate, thionyl chloride and acetic anhydride were purchased from Aldrich and used as received.

DMPU (1,3-Dimethyl-3,4,5,6-tetrahydro-2(1H)-pyrimidinone, absolute, over molecular sieve $\left(\mathrm{H}_{2} \mathrm{O} \leq 0.03 \%\right), \geq 99.0 \%(\mathrm{GC})$ was purchased from Aldrich and sparged with nitrogen after use for $\sim 30$ seconds. Although sequential drying of DMPU over $4 \AA$ molecular sieve before use could reduce water content to as low as $16 \mathrm{ppm}$, successful reactions were observed in DMPU containing as much as 1000 ppm water. All other dry solvents were prepared from ACS grade, inhibitor free solvents by passage through activated alumina and molecular sieves in a Vacuum Atmospheres solvent purification system. Water content was routinely measured using KarlFisher titration (Metrohm) and was less than $30 \mathrm{ppm}$ in all cases.

Iodobenzene, 4'-iodoacetophenone, 4-iodoanisole, 2-bromotoluene, 4-iodobenzonitrile, ethyl-4-bromobutyrate, 4-iodobenzoic acid, 3-bromopropylamine hydrobromide, 5-bromoindole, 2-bromoheptane (technical grade), 5-bromo-2-methyl-2-pentene, and 4-bromophenylboronic acid were purchased from Aldrich and used without further purification. 4-iodobenzotrifluoride, and neo-pentyliodide, were purchased from Aldrich and filtered through a short pad of activated basic alumina $(1.5 \mathrm{~cm})$ under nitrogen before use. 3-bromopropyl-carbamic acid tert-butyl ester [83948-53-2], (3-bromopropyl)carbamic acid benzyl ester [39945-54-5], ${ }^{3} \quad p$ bromophenylboronic pinacol ester, ${ }^{4}$ and $\mathrm{N}$-acetyl-5-bromoindole ${ }^{5}$ were synthesized according to the literature procedures and spectral data were in agreement with the reported values. Organic iodides that had been stored on the bench for long periods of time did not always provide consistently high results. In these cases, filtration of the organic iodide through basic alumina or silica gel provided material that could again be coupled in high yield.

\section{Methods.}

NMR chemical shifts are reported in ppm and referenced to the residual solvent peak $\mathrm{CDCl}_{3}\left(\delta=7.26 \mathrm{ppm}{ }^{1} \mathrm{H}\right.$ or $\left.\delta=77.16 \mathrm{ppm}{ }^{13} \mathrm{C}\right)$ as an internal standard and trifluorotoluene $(\delta=$ $0.000 \mathrm{ppm})$ as an external standard $\left({ }^{19} \mathrm{~F}\right)$. NMR spectra were recorded on Bruker model Avance NMR spectrometer operating at $400.13 \mathrm{MHz}$ or $500.13 \mathrm{MHz}$ proton NMR frequency, and data analysis was performed using the iNMR software package (version 3.1.5).

GC analyses were performed on an Agilent 7890A GC equipped with dual DB-5 columns (20 $\mathrm{m} \times 180 \mu \mathrm{m} \times 0.18 \mu \mathrm{m})$, dual FID detectors and using hydrogen as the carrier gas. The 
analysis method used in all cases was $1 \mu \mathrm{L}$ inj. of sample, inj. temp of $300{ }^{\circ} \mathrm{C}, 100: 1$ split ratio, initial inlet pressure was 20.3 psi but varied as the column flow was held constant at $1.8 \mathrm{~mL} / \mathrm{min}$ for the duration of the run. Initial oven temperature of $50{ }^{\circ} \mathrm{C}$ was held for $0.46 \mathrm{~min}$ followed by a temperature ramp up to $300{ }^{\circ} \mathrm{C}$ at $65^{\circ} \mathrm{C} / \mathrm{min}$ and finally the temperature was held at $300{ }^{\circ} \mathrm{C}$ for 0.69 min. Total run time was $\sim 5$ min. FID temperature was $325^{\circ} \mathrm{C}$.

GC/MS analyses were performed on a Shimadzu GCMS-QP2010 equipped with an RTXXLB column ( $30 \mathrm{~m} \times 0.25 \mathrm{~mm} \times 0.28 \mu \mathrm{m})$ with a quadrupole mass analyzer using helium as the carrier gas. The analysis method used in all cases was $5 \mu \mathrm{L}$ inj. of sample, inj. temp of $225{ }^{\circ} \mathrm{C}$, 25:1 split ratio, initial inlet pressure was $7.8 \mathrm{psi}$, but varied as the column flow was held constant at $1.0 \mathrm{~mL} / \mathrm{min}$ for the duration of the run, the interface temperature was held at $250{ }^{\circ} \mathrm{C}$, and the ion source $(\mathrm{EI}, 30 \mathrm{eV})$ was held at $250{ }^{\circ} \mathrm{C}$. Initial oven temperature was held at $50{ }^{\circ} \mathrm{C}$ for $3 \mathrm{~min}$ with the detector off followed by a temperature ramp, with the detector on, to $280{ }^{\circ} \mathrm{C}$ at 40 ${ }^{\circ} \mathrm{C} / \mathrm{min}$, and finally the temperature was held at $280{ }^{\circ} \mathrm{C}$ for $3 \mathrm{~min}$. Total run time was $11.75 \mathrm{~min}$.

Chromatography was performed on silica gel (EMD, silica gel 60, particle size 0.040$0.063 \mathrm{~mm}$ ) using standard flash techniques or on a Biotage Isolera One (KP-SIL $50 \mathrm{~g}$ Biotage snap cartridge with samplet, $40 \mathrm{~mL} / \mathrm{min}$ flow rate, detection at $254 \mathrm{~nm}$ and $280 \mathrm{~nm}$ ). Products were visualized by one of the following methods: UV stain, ninhydrin stain, $\mathrm{KMnO}_{4}$ stain or by GC. $\mathrm{NJ})$.

Elemental Analyses were performed by Robertson Microlit Laboratories, Inc. (Madison, 


\section{Optimization and Control Reactions}

Typical procedure for optimization screens and control reactions. Reactions were set up in a nitrogen filled glove box. To an oven-dried 1-dram vial containing a teflon-coated stir-bar was added the required amount of appropriate catalyst and ligand(s) followed by DMPU ( $2 \mathrm{~mL})$, pyridine (if used), organohalide(s) $\left(0.500 \mathrm{mmol}\right.$ each), $\mathrm{Mn}^{0}$ (2.00 equiv), and dodecane $(10.0 \mu \mathrm{L}$ internal standard). The reaction vials were capped with a PTFE-faced silicone septum, removed from the glove box and heated in a reaction block on the benchtop. After 15-24 h reaction time, $50 \mu \mathrm{L}$ aliquots of reaction mixture were removed with a $50 \mu \mathrm{L}$ gas-tight syringe and quenched with $50 \mu \mathrm{L}$ of $1 \mathrm{M}$ aqueous $\mathrm{NaHSO}_{4}$, diluted with ethyl ether or ethyl acetate $(1 \mathrm{~mL})$, and filtered through a short silica pad $(1.5 \mathrm{~cm})$ in a pipette packed with glass wool. The filtrate was analyzed by gas chromatography and percent yield or percent conversion based on unreacted starting material were calculated.

Table S1: Direct Insertion of $\mathrm{Mn}^{0}$.

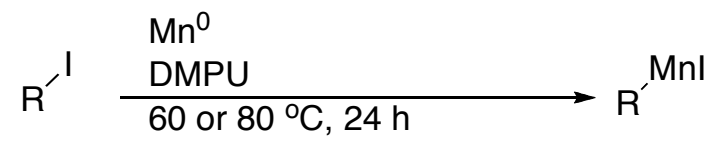

\begin{tabular}{lll}
$\mathrm{R}=\mathrm{C}_{8} \mathrm{H}_{17}$ or $\mathrm{Ph}$ & & \\
\hline Substrate & $\mathrm{T}\left({ }^{\circ} \mathrm{C}\right)$ & Consumption of RI $(\%)^{a, b}$ \\
\hline $\mathrm{C}_{8} \mathrm{H}_{17}-\mathrm{I}$ & 60 & 0 \\
$\mathrm{C}_{8} \mathrm{H}_{17}-\mathrm{I}$ & 80 & 19 \\
$\mathrm{Ph}-\mathrm{I}$ & 60 & 0 \\
$\mathrm{Ph}-\mathrm{I}$ & 80 & 0 \\
\hline
\end{tabular}
${ }^{a}$ Based on unreacted iodide (corrected vs. dodecane). ${ }^{b}$ Catalytic cross coupling of Ph-I and
$\mathrm{C}_{8} \mathrm{H}_{17}-\mathrm{I}$ is complete in 3 hours

Table S2: Direct Insertion of $\mathrm{Mn}^{0}$ in the Presence of Pyridine.

$$
\begin{aligned}
& \mathrm{R}^{\prime \prime} \quad \begin{array}{l}
\mathrm{Mn}^{0} \\
\frac{\mathrm{DMPU} \text { mol\% pyridine }}{60 \text { or } 80^{\circ} \mathrm{C}, 24 \mathrm{~h}}
\end{array} \\
& \mathrm{R}=\mathrm{R}_{8} \mathrm{H}_{17} \text { or } \mathrm{Ph}
\end{aligned}
$$

\begin{tabular}{lll}
\hline Substrate & $\mathrm{T}\left({ }^{\circ} \mathrm{C}\right)$ & Consumption of RI $(\%)^{a, b}$ \\
\hline $\mathrm{C}_{8} \mathrm{H}_{17}-\mathrm{I}$ & 60 & 9 \\
$\mathrm{C}_{8} \mathrm{H}_{17}-\mathrm{I}$ & 80 & 59 \\
$\mathrm{Ph}-\mathrm{I}$ & 60 & 0 \\
$\mathrm{Ph}-\mathrm{I}$ & 80 & 0 \\
\hline
\end{tabular}

${ }^{a}$ Based on unreacted iodide (corrected vs. dodecane). ${ }^{b}$ Catalytic cross coupling of $\mathrm{Ph}-\mathrm{I}$ and $\mathrm{C}_{8} \mathrm{H}_{17}-\mathrm{I}$ is complete in 3 hours 
Table S3: Solvent Effect.

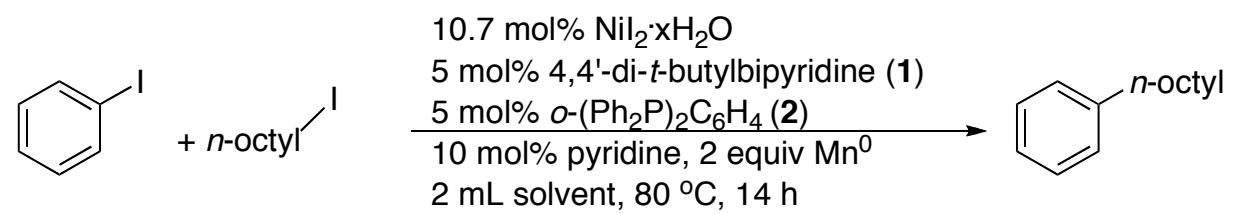

\begin{tabular}{ll}
\hline Solvent & ${\text { Yield }(\%)^{a}}^{a}$ \\
\hline DMPU & 87 \\
DMAc & 71 \\
NMP & 64 \\
DMF & 60 \\
DMI & 65 \\
THF & 37 \\
Toluene & trace \\
DCM & trace \\
DMSO & 19
\end{tabular}

${ }^{a}$ GC yield corrected $v s$. dodecane internal standard after $14 \mathrm{~h}$ reaction time.

Reaction is complete in $14 \mathrm{~h}$.

Table S4: GC Product Distribution Data for Substrates 3a-p

\begin{tabular}{|c|c|c|c|c|c|c|c|}
\hline Entry & Product & $\begin{array}{l}\mathbf{3}^{a} \\
(\% \text { yield })\end{array}$ & $\begin{array}{l}3^{b} \\
(\mathrm{~A} \%)\end{array}$ & $\begin{array}{l}\text { Biaryl }^{b} \\
(\mathrm{~A} \%)\end{array}$ & $\begin{array}{l}\beta \text {-Hydride } \\
\text { Elimination }^{b} \\
(\mathrm{~A} \%)\end{array}$ & $\begin{array}{l}\text { Bialkyl }^{b} \\
(\mathrm{~A} \%)\end{array}$ & $\begin{array}{l}\text { Reduced Arene } \\
(\mathrm{A} \%)\end{array}$ \\
\hline 1 & $3 a$ & 77 & 82 & 4 & 5 & 0 & 4 \\
\hline 2 & $3 b$ & 75 & 75 & 7 & 6 & 1 & 1 \\
\hline 3 & $3 c$ & 75 & 63 & 11 & 2 & 0.5 & 2 \\
\hline 4 & 3d & 69 & 72 & 7 & 2 & 0.5 & 5 \\
\hline $5^{d}$ & $3 e$ & 55 & 48 & $\mathrm{ND}^{c}$ & 6 & 0 & $\mathrm{ND}^{c}$ \\
\hline 6 & $3 f$ & 82 & 88 & $\mathrm{ND}^{c}$ & 2 & 1 & $\mathrm{ND}^{c}$ \\
\hline 7 & $3 g$ & 82 & 63 & $\mathrm{ND}^{c}$ & 4 & 2 & $\mathrm{ND}^{c}$ \\
\hline 8 & $3 h$ & 78 & 73 & $\mathrm{ND}^{c}$ & 0 & 3 & $\mathrm{ND}^{c}$ \\
\hline 9 & $3 \mathbf{i}$ & 77 & 91 & $\mathrm{ND}^{c}$ & 0 & 0.5 & $\mathrm{ND}^{c}$ \\
\hline 10 & $3 \mathbf{j}$ & 77 & 61 & 6 & 4 & 18 & 5 \\
\hline 11 & $3 \mathbf{k}$ & 85 & 87 & 3 & 3 & 1 & 2 \\
\hline 12 & 31 & 65 & 44 & 5 & $\mathrm{ND}^{c}$ & 13 & 2 \\
\hline 13 & $3 m$ & 64 & 62 & 12 & $\mathrm{ND}^{c}$ & 6 & 2 \\
\hline 14 & $3 n$ & 75 & 73 & $\mathrm{ND}^{c}$ & 0 & 6 & 3 \\
\hline 15 & 30 & 38 & 56 & 19 & 0 & 0 & 3 \\
\hline 16 & $3 p$ & 88 & 64 & 0.5 & 5 & 3 & 5 \\
\hline
\end{tabular}

${ }^{a}$ Isolated yield of purified product, average of two runs. ${ }^{b}$ Area percent determined by GC from crude reaction mixtures, average of two runs. In cases where the $\mathrm{A} \%$ is 0 , a peak of $<0.5 \mathrm{~A} \%$ is present or no peak is observed and the retention time of the compound is known. ${ }^{c}$ Not detected by GC analysis. No assignable peak and retention time of compound is unknown. ${ }^{d}$ Data from run 1 only, no final data point collected for run 2. 
Table S5: Substrate scope using a single ligand (1) versus a combination of ligands $\mathbf{1}$ and $\mathbf{2} .^{a}$

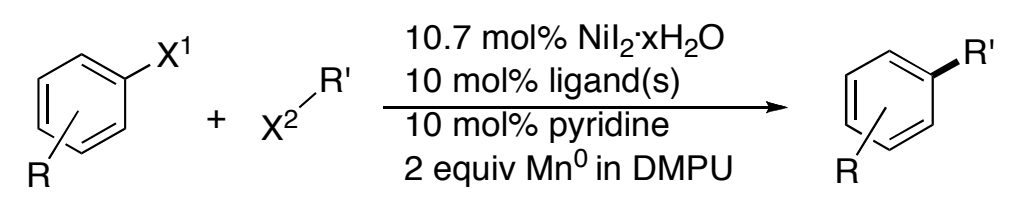
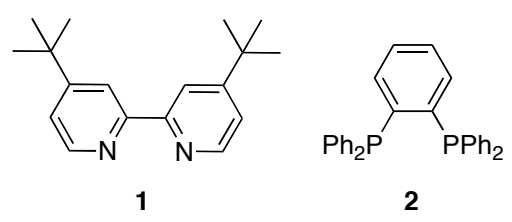

\begin{tabular}{|c|c|c|c|c|c|c|c|}
\hline Entry & Product & & $X^{1}$ & $X^{2}$ & $\begin{array}{c}\mathrm{T} \\
\left({ }^{\circ} \mathrm{C}\right)\end{array}$ & $\begin{array}{l}\text { Yield }(\%)^{b} \\
\text { Ligand (1) }\end{array}$ & $\begin{array}{c}\text { Yield (\%) } \\
\text { Ligands (1) and (2) }\end{array}$ \\
\hline 1 & $\mathrm{R}=\mathrm{OMe}$ & $3 b$ & I & I & 80 & 70 & 75 \\
\hline 2 & $\mathrm{R}=\mathrm{CF}_{3}$ & $3 c$ & I & I & 80 & 60 & 75 \\
\hline 3 & $\mathrm{R}=\mathrm{C}(\mathrm{O}) \mathrm{Me}$ & 3d & I & I & 80 & 54 & 69 \\
\hline 4 & & $3 f$ & $\mathrm{Br}$ & I & 60 & 74 & 82 \\
\hline 5 & & $3 g$ & $\mathrm{Br}$ & I & 80 & 62 & 82 \\
\hline 6 & & $3 \mathbf{i}$ & $\mathrm{Br}$ & I & 60 & 83 & 77 \\
\hline $7^{d}$ & & $3 \mathbf{j}$ & I & $\mathrm{Br}$ & 80 & 57 & 64 \\
\hline 8 & & 30 & I & I & 80 & 29 & 38 \\
\hline
\end{tabular}

\footnotetext{
${ }^{a}$ Organohalides (1.00 mmol each), $0.107 \mathrm{mmol} \mathrm{NiI}{ }_{2} \bullet \mathrm{xH}_{2} \mathrm{O}, 0.100 \mathrm{mmol}$ ligand(s), $0.099 \mathrm{mmol}$ pyridine, $2.00 \mathrm{mmol} \mathrm{M{ } ^ { 0 }}$ powder, and $4 \mathrm{~mL}$ of DMPU are heated for 3-21 hours, ${ }^{b}$ Isolated yield of purified product using only $4,4^{\prime}$-di-t-butylbipyridine $1(0.100 \mathrm{mmol}),{ }^{c}$ Isolated yield of purified product using $0.0499 \mathrm{mmol}$ 4,4'-di-t-butylbipyridine (1) and $0.0499 \mathrm{mmol} 1,2-$ Bis(diphenylphosphino)benzene (2). Average of two runs, ${ }^{d}$ Longer reaction time required (37 h).
} 


\section{Preparation of Organohalides}<smiles>OCc1ccc(I)cc1</smiles>

4-iodo(phenyl)methanol [CAS: 18282-51-4]. ${ }^{6}$ To a stirred suspension of 4-iodobenzoic acid (5.00 g, $20.0 \mathrm{mmol}, 1.00$ equiv) in dichloromethane $(65 \mathrm{~mL})$ was added thionyl chloride $(13 \mathrm{~mL}$, $179 \mathrm{mmol}, 9.0$ equiv) before heating to $70{ }^{\circ} \mathrm{C}$ overnight. The resulting pale yellow solution was evaporated to dryness under reduced pressure to afford a pale yellow solid. The pale yellow solid, 4-iodobenzoyl chloride, was dissolved in $30 \mathrm{~mL}$ dioxane, and added dropwise to a stirred suspension of sodium borohydride $\left(1.5 \mathrm{~g}, 40 \mathrm{mmol}, 2.0\right.$ equiv) in dioxane $(30 \mathrm{~mL})$ at $0{ }^{\circ} \mathrm{C}$ over $25 \mathrm{~min}$. The resulting mixture was heated to $100{ }^{\circ} \mathrm{C}$ for $90 \mathrm{~min}$, then cooled to room temperature. Finally, $\mathrm{H}_{2} \mathrm{O}(30 \mathrm{~mL})$ was added slowly. Caution: Evolves Hydrogen Gas! The mixture was extracted with dichloromethane $(3 \times 50 \mathrm{~mL})$. Organic layers were combined, washed with $\mathrm{H}_{2} \mathrm{O}(2 \times 50 \mathrm{~mL}), \mathrm{HCl}(1 \mathrm{~N}, 2 \times 50 \mathrm{~mL}), \mathrm{NaOH}(1 \mathrm{~N}, 2 \times 50 \mathrm{~mL}), \mathrm{H}_{2} \mathrm{O}(1 \times 50 \mathrm{~mL})$, and brine $(1 \times 50 \mathrm{~mL})$, dried over $\mathrm{MgSO}_{4}$, filtered and evaporated under reduced pressure to give a white powder (4.54 g, 97\% two steps).

${ }^{1} \mathrm{H}$ NMR $\left(400 \mathrm{MHz}, \mathrm{CDCl}_{3}\right) \delta 7.69(\mathrm{~d}, J=8.3 \mathrm{~Hz}, 2 \mathrm{H}), 7.11(\mathrm{~d}, J=8.3 \mathrm{~Hz}, 2 \mathrm{H}), 4.64(\mathrm{~d}$, $J=5.2 \mathrm{~Hz}, 2 \mathrm{H}), 1.73(\mathrm{t}, J=5.5 \mathrm{~Hz}, 1 \mathrm{H})$.

${ }^{13} \mathrm{C} \mathrm{NMR}\left(100 \mathrm{MHz}, \mathrm{CDCl}_{3}\right) \delta$ 140.6, 137.8, 128.9, 93.1, 64.8.

GC-MS $m / z$ (\% relative intensity, ion): $234.85\left(1.900, \mathrm{M}^{+}\right), 107.05\left(31.30, \mathrm{M}^{+}-\mathrm{I}\right), 79.00$ (100.0, $\left.\mathrm{M}^{+}-\mathrm{CHIO}\right)$.

NOTE: Long-term storage of 4-iodo(phenyl)methanol on benchtop resulted in diminished yields of reductive coupling reactions with the reagent. Purification of the compound immediately before use helped to observe reproducible results with reductive coupling reactions (Table 1, entry 8). The product could be purified passing through a short silica gel column (hexanes: ethyl acetate $80: 20)$.

\section{Reductive Cross-Coupling Reactions General procedure for preparative reactions (Table 1).}

On the benchtop, $\mathrm{NiI}_{2} \mathrm{xH}_{2} \mathrm{O}(\mathrm{x}=3.5, \mathrm{MW}=375.56,40.3 \mathrm{mg}, 0.107 \mathrm{mmol}, 0.107$ equiv $)$, 1,2-bis(diphenylphosphino)benzene $(22.3 \mathrm{mg}, 0.0499 \mathrm{mmol}, 0.0499$ equiv), 4,4'-di-tert-butyl2,2'-dipyridyl (13.4 mg, $0.0499 \mathrm{mmol}, 0.0499$ equiv), and solid substrates (1.00 mmol, 1.00 equiv) were weighed on weigh paper and transferred to a flame dried $10 \mathrm{~mL}$ Schlenk flask equipped with a magnetic stir bar. The flask was then capped with a rubber septum and purged with $\mathrm{N}_{2}$ gas $(\sim 30 \mathrm{sec}$.). DMPU (4 mL), pyridine $(8.0 \mu \mathrm{L}, 0.099 \mathrm{mmol}, 0.099$ equiv), and liquid substrates (1.00 mmol, 1.00 equiv) were injected into the flask. The generally deep purple solution was sparged with $\mathrm{N}_{2}$ gas ( $30-60 \mathrm{sec}$.), and manganese powder $(110 \mathrm{mg}, 2.00 \mathrm{mmol}$, 2.00 equiv) was added. The reaction flask was placed in a 60 or $80^{\circ} \mathrm{C}$ oil bath and stirred under positive pressure of nitrogen.

The reaction progress was followed by GC analysis. At appropriate times $50 \mu \mathrm{L}$ aliquots of reaction mixture were removed with a $50 \mu \mathrm{L}$ gas-tight syringe and quenched with $50 \mu \mathrm{L}$ of 1 $\mathrm{M}$ aqueous $\mathrm{NaHSO}_{4}$, diluted with ethyl ether or ethyl acetate $(1 \mathrm{~mL})$, and filtered through a short 
silica pad $(1.5 \mathrm{~cm})$ in a pipette packed with glass wool. The filtrate was analyzed by gas chromatography.

The reactions generally turn from orange/brown to black when complete (Figure S1). Upon completion (judged as $<1$ Area \% of starting materials remaining by GC analysis) the reaction mixture was filtered though a short plug of silica gel to remove metal salts and DMPU, eluted with diethyl ether, and concentrated under reduced pressure followed by purification on a silica gel. Alternatively, the entire reaction mixture was loaded onto a silica gel column for purification. Column fractions were analyzed by GC for compounds 3a, 3l-3p and by TLC for compounds $\mathbf{3 b}-\mathbf{3 k}$. The compounds on TLC were visualized using $\mathrm{KMnO}_{4}$ stain for $\mathbf{3 g}$ and $\mathbf{3 h}$, ninhydrin stain for $\mathbf{3 k}$ and iodine for compounds $\mathbf{3 b} \mathbf{b} \mathbf{3} \mathbf{f}, \mathbf{3 i}$, and $\mathbf{3 j}$.

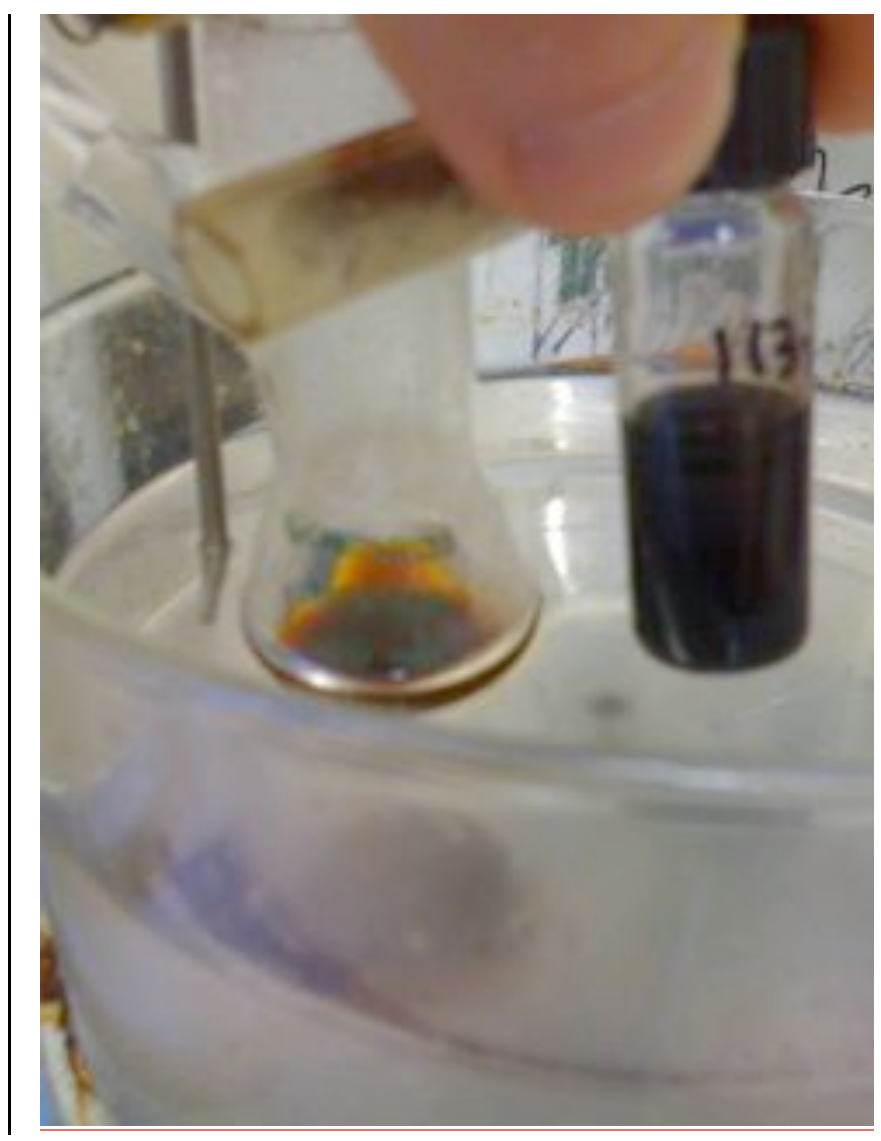

Figure S1. Reaction completion is accompanied by color change from orange (left) to black (right). 


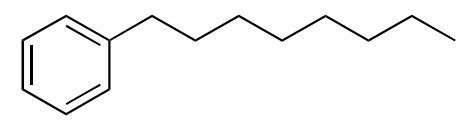

1-octylbenzene (3a) (Table 1, entry 1) [CAS: 2189-60-8]. ${ }^{6}$ The general procedure was followed with iodobenzene $(112 \mu \mathrm{L}, 1.00 \mathrm{mmol}, 1.00$ equiv) and 1-iodooctane $(181 \mu \mathrm{L}, 1.00$ mmol, 1.00 equiv) at $80{ }^{\circ} \mathrm{C}$ for $3 \mathrm{~h}$. The product was isolated by chromatography (pentane) as a colorless oil. First run $146 \mathrm{mg}(77 \%)$. Second run $146 \mathrm{mg}(77 \%)$.

${ }^{1} \mathrm{H}$ NMR $\left(500 \mathrm{MHz}, \mathrm{CDCl}_{3}\right) \delta$ 7.36-7.23 (m, 5H), $2.68(\mathrm{t}, J=8.4 \mathrm{~Hz}, 2 \mathrm{H}), 1.72-1.66(\mathrm{~m}$, 2H), 1.39-1.35 (m, 10H), $0.97(\mathrm{t}, J=6.6 \mathrm{~Hz}, 3 \mathrm{H})$.

${ }^{13} \mathrm{C}$ NMR $\left(125 \mathrm{MHz}, \mathrm{CDCl}_{3}\right) \delta$ 143.0, 128.5, 128.4, 125.7, 36.2, 32.1, 31.7, 29.7, 29.6, $29.5,22.9,14.3$.

GC-MS $m / z$ GC-MS $m / z$ (\% relative intensity, ion): $190.15\left(14.30, \mathrm{M}^{+}\right), 92.00$ (100.0, $\left.\mathrm{M}^{++-} \mathrm{C}_{7} \mathrm{H}_{14}\right), 91.00\left(67.20, \mathrm{M}^{+}-\mathrm{C}_{7} \mathrm{H}_{15}\right)$<smiles>CCCCCCCCc1ccc(OC)cc1</smiles>

1-methoxy-4-octylbenzene (3b) (Table 1, entry 2) [CAS: 3307-19-5]. ${ }^{7}$ The general procedure was followed with 4-iodoanisole $(234 \mathrm{mg}, 1.00 \mathrm{mmol}, 1.00$ equiv) and 1-iodooctane $(181 \mu \mathrm{L}$, $1.00 \mathrm{mmol}, 1.00$ equiv) at $80{ }^{\circ} \mathrm{C}$ for $15 \mathrm{~h}$. The product was isolated by chromatography (90:10 pentane:ether) as colorless oil. First run $170 \mathrm{mg}$ (77\%). Second run $160 \mathrm{mg}(72 \%)$.

${ }^{1} \mathrm{H}$ NMR $\left(500 \mathrm{MHz}, \mathrm{CDCl}_{3}\right) \delta 7.09(\mathrm{~d}, J=8.6 \mathrm{~Hz}, 2 \mathrm{H}), 6.83(\mathrm{~d}, J=8.6 \mathrm{~Hz}, 2 \mathrm{H}), 3.79$ (s, $3 \mathrm{H}), 2.55(\mathrm{t}, J=7.5 \mathrm{~Hz}, 2 \mathrm{H}), 1.62-1.55(\mathrm{~m}, 2 \mathrm{H}), 1.33-1.28(\mathrm{~m}, 10 \mathrm{H}), 0.89(\mathrm{t}, J=6.7 \mathrm{~Hz}, 3 \mathrm{H})$. ${ }^{13} \mathrm{C} \mathrm{NMR}\left(125 \mathrm{MHz}, \mathrm{CDCl}_{3}\right) \delta$ 157.8, 135.2, 129.4, 113.8, 55.4, 35.2, 32.1, 31.9, 29.9, 29.7, 29.4, 22.8, 14.3.

GC-MS $m / z$ (\% relative intensity, ion): $220.10\left(10.70, \mathrm{M}^{+}\right), 121.05\left(100.0, \mathrm{M}^{+}-\mathrm{C}_{7} \mathrm{H}_{15}\right)$.<smiles>CCCCCCCCc1ccc(C(F)(F)F)cc1</smiles>

1-(trifluoromethyl)-4-octylbenzene. (3c) (Table 1, entry 3). The general procedure was followed with 4-iodobenzotrifluoride $(147 \mu \mathrm{L}, 1.00 \mathrm{mmol}, 1.00$ equiv) and 1-iodooctane (181 $\mu \mathrm{L}, 1.00 \mathrm{mmol}, 1.00$ equiv) at $80^{\circ} \mathrm{C}$ for $19 \mathrm{~h}$. The product was isolated by chromatography (hexanes) as pale yellow oil. First run $180 \mathrm{mg}$ (70\%). Second run $204 \mathrm{mg}$ (79\%).

${ }^{1} \mathrm{H}$ NMR $\left(500 \mathrm{MHz}, \mathrm{CDCl}_{3}\right) \delta 7.55(\mathrm{~d}, J=8.1 \mathrm{~Hz}, 2 \mathrm{H}), 7.30(\mathrm{~d}, J=8.1 \mathrm{~Hz}, 2 \mathrm{H}), 2.68(\mathrm{t}$, $J=7.6 \mathrm{~Hz}, 2 \mathrm{H}), 1.68-1.62(\mathrm{~m}, 2 \mathrm{H}), 1.34-1.30(\mathrm{~m}, 10 \mathrm{H}), 0.91(\mathrm{t}, J=6.8 \mathrm{~Hz}, 3 \mathrm{H})$

${ }^{13} \mathrm{C} \mathrm{NMR}\left(125 \mathrm{MHz}, \mathrm{CDCl}_{3}\right) \delta 147.2,128.8,128.4$ (q, $\left.J=32.0 \mathrm{~Hz}\right), 125.3$ (q, $\left.J=3.8 \mathrm{~Hz}\right)$, $124.6(\mathrm{q}, J=217.7 \mathrm{~Hz}), 36.0,32.1,31.4,29.9,29.6,29.4,22.9,14.2$.

${ }^{19} \mathrm{~F}$ NMR $\left(375 \mathrm{MHz}, \mathrm{C}_{6} \mathrm{D}_{6}\right) \delta 0.567$

GC-MS $m / z$ (\% relative intensity, ion): $258.05\left(19.90, \mathrm{M}^{+}\right), 160.15\left(100.0, \mathrm{M}^{+}-\mathrm{C}_{7} \mathrm{H}_{14}\right)$, 91.00 (19.80, $\left.\mathrm{M}^{+}-\mathrm{C}_{8} \mathrm{H}_{15} \mathrm{~F}_{3}\right)$.<smiles>CCCCCCCCc1ccc(C(C)=O)cc1</smiles>

1-(4-octylphenyl)ethanone (3d) (Table 1, entry 4) [CAS: 10541-56-7]. ${ }^{8}$ The general procedure was followed and the reaction was run with 4 '-iodoacetophenone $(246 \mathrm{mg}, 1.00 \mathrm{mmol}, 1.00$ 
equiv) and 1-iodooctane $\left(181 \mu \mathrm{L}, 1.00 \mathrm{mmol}, 1.00\right.$ equiv) at $80^{\circ} \mathrm{C}$ for $8 \mathrm{~h}$. The product was isolated by chromatography (80:20 pentane:ether) as pale yellow oil. First run $158 \mathrm{mg}(68 \%)$. Second run $159 \mathrm{mg}(69 \%)$.

${ }^{1} \mathrm{H}$ NMR $\left(500 \mathrm{MHz}, \mathrm{CDCl}_{3}\right) \delta 7.88(\mathrm{~d}, J=8.2 \mathrm{~Hz}, 2 \mathrm{H}), 7.26(\mathrm{~d}, J=8.2 \mathrm{~Hz}, 2 \mathrm{H}), 2.66(\mathrm{t}$, $J=7.5 \mathrm{~Hz}, 2 \mathrm{H}), 2.58 \mathrm{~s},(3 \mathrm{H}), 1.62-1.59(\mathrm{~m}, 2 \mathrm{H}),, 1.31-1.26(\mathrm{~m}, 10 \mathrm{H}), 0.88(\mathrm{t}, J=6.6 \mathrm{~Hz}, 3 \mathrm{H})$.

${ }^{13} \mathrm{C} \mathrm{NMR}\left(125 \mathrm{MHz}, \mathrm{CDCl}_{3}\right) \delta 197.9,148.9,135.0,128.7,128.6,36.1,32.0,31.2,29.5$, 29.4, 29.3, 26.6, 22.8, 14.2.

GC-MS $m / z$ (\% relative intensity, ion): $232.05\left(16.90, \mathrm{M}^{+}\right), 217.05\left(100.0, \mathrm{M}^{+}-\mathrm{CH}_{3}\right)$, $105.00\left(17.70, \mathrm{M}^{+}-\mathrm{C}_{9} \mathrm{H}_{20}\right), 91.00\left(23.40, \mathrm{M}^{+}-\mathrm{C}_{9} \mathrm{H}_{15} \mathrm{O}\right)$.

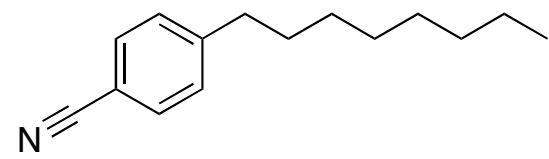

4-octylbenzonitrile (3e) (Table 1, entry 5). ${ }^{9}$ The general procedure was followed with 4iodobenzonitrile $(229 \mathrm{mg}, 1.00 \mathrm{mmol}, 1.00$ equiv) and 1 -iodooctane $(181 \mu \mathrm{L}, 1.00 \mathrm{mmol}, 1.00$ equiv) at $80^{\circ} \mathrm{C}$ for $15 \mathrm{~h}$. The product was isolated by chromatography (80:20 pentane:ether, first run and 90:10 pentane:ether, second run) as faintly yellow oil. First run $138 \mathrm{mg}$ (64\%). Second run $101 \mathrm{mg}$ (47\%, reaction did not proceed to completion).

${ }^{1} \mathrm{H}$ NMR $\left(500 \mathrm{MHz}, \mathrm{CDCl}_{3}\right) \delta 7.54(\mathrm{~d}, J=8.2 \mathrm{~Hz}, 2 \mathrm{H}), 7.26(\mathrm{~d}, J=8.1 \mathrm{~Hz}, 2 \mathrm{H}), 2.65(\mathrm{t}, J$ $=7.6 \mathrm{~Hz}, 2 \mathrm{H}), 1.64-1.57(\mathrm{~m}, 2 \mathrm{H}), 1.30-1.25(\mathrm{~m}, 10 \mathrm{H}), 0.87(\mathrm{t}, J=5.5 \mathrm{~Hz}, 3 \mathrm{H})$.

${ }^{13} \mathrm{C} \mathrm{NMR}\left(125 \mathrm{MHz}, \mathrm{CDCl}_{3}\right) \delta$ 148.7, 132.1, 129.3, 119.2, 109.6, 36.2, 31.9, 31.0, 29.8, $29.4,29.3,22.7,14.2$.

GC-MS $m / z$ (\% relative intensity, ion): $215.05\left(14.30, \mathrm{M}^{+}\right), 117.05\left(100.0, \mathrm{M}^{+}-\mathrm{C}_{7} \mathrm{H}_{14}\right)$.

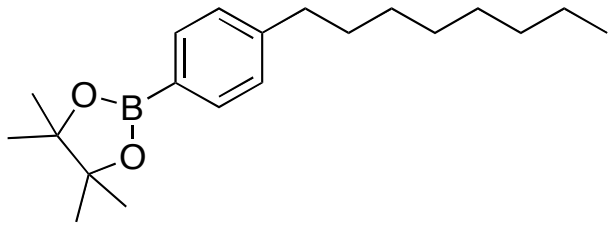

4,4,5,5-tetramethyl-2-2(4-octylphenyl)-1,2,3-dioxaborolane (3f) (Table 1, entry 6). The general procedure was followed with $p$-bromophenylboronic pinacol ester $(283 \mathrm{mg}, 1.00 \mathrm{mmol}$, 1.00 equiv) and 1-iodooctane $\left(181 \mu \mathrm{L}, 1.00 \mathrm{mmol}, 1.00\right.$ equiv) at $60{ }^{\circ} \mathrm{C}$ for $16 \mathrm{~h}$. The product was isolated by chromatography (hexanes: tert-butyl methyl ether 95: 5) as yellow oil. First run $271 \mathrm{mg}(86 \%)$. Second run $245 \mathrm{mg}(78 \%)$.

${ }^{1} \mathrm{H}$ NMR $\left(500 \mathrm{MHz}, \mathrm{CDCl}_{3}\right) \delta 7.82(\mathrm{~d}, J=7.9 \mathrm{~Hz}, 2 \mathrm{H}), 7.28(\mathrm{~d}, J=7.6 \mathrm{~Hz}, 2 \mathrm{H}), 2.70(\mathrm{t}$, $J=7.5 \mathrm{~Hz}, 2 \mathrm{H}), 1.73-1.68(\mathrm{~m}, 2 \mathrm{H}), 1.43-1.35(\mathrm{~m}, 22 \mathrm{H}), 0.97(\mathrm{t}, J=7.0 \mathrm{~Hz}, 3 \mathrm{H})$.

${ }^{13} \mathrm{C} \mathrm{NMR}\left(125 \mathrm{MHz}, \mathrm{CDCl}_{3}\right) \delta 146.5,134.9,128.0,83.7,36.3,32.0,31.5,29.9,29.6$, 29.5, 29.4, 25.0, 22.8, 14.2 .

GC-MS $m / z$ (\% relative intensity, ion): $316.20\left(38.50, \mathrm{M}^{+}\right), 301.20\left(37.70, \mathrm{M}^{+}-\mathrm{CH}_{3}\right)$, $230.10\left(100.0, \mathrm{M}^{+}-\mathrm{C}_{5} \mathrm{H}_{11} \mathrm{O}\right), 217.05\left(63.29, \mathrm{M}^{+}-\mathrm{C}_{7} \mathrm{H}_{15}\right)$.

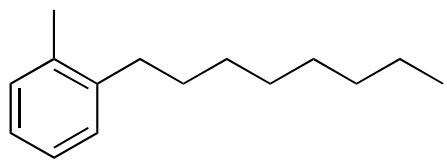

1-methyl-2-octylbenzene (3g) (Table 1, entry 7) [CAS: 79835-99-7]. The general procedure was followed with 2-bromotoluene $(120 \mu \mathrm{L}, 1.00 \mathrm{mmol}, 1.00$ equiv) and 1-iodooctane $(181 \mu \mathrm{L}$, 
$1.00 \mathrm{mmol}, 1.00$ equiv) at $80{ }^{\circ} \mathrm{C}$ for $15 \mathrm{~h}$. The product was isolated by chromatography (pentane) as colorless oil. First run $162 \mathrm{mg}$ (79\%). Second run $169 \mathrm{mg}(83 \%)$.

${ }^{1} \mathrm{H}$ NMR $\left(500 \mathrm{MHz}, \mathrm{CDCl}_{3}\right) \delta$ 7.13-7.11 (m, 4H), $2.59(\mathrm{t}, J=7.8 \mathrm{~Hz}, 2 \mathrm{H}), 2.31(\mathrm{~s}, 3 \mathrm{H})$, $1.60-1.53(\mathrm{~m}, 2 \mathrm{H}), 1.39-1.28(\mathrm{~m}, 10 \mathrm{H}), 0.89(\mathrm{t}, J=6.6 \mathrm{~Hz}, 3 \mathrm{H})$.

${ }^{13} \mathrm{C}$ NMR $\left(125 \mathrm{MHz}, \mathrm{CDCl}_{3}\right) \delta 141.3,135.9,130.2,128.9,126.0,125.8,33.5,32.1,30.5$, 29.9, 29.7, 29.5, 22.9, 19.4, 14.3.

GC-MS $m / z$ (\% relative intensity, ion): $204.10\left(11.90, \mathrm{M}^{+}\right), 105.05\left(100.0, \mathrm{M}^{+}-\mathrm{C}_{7} \mathrm{H}_{15}\right)$, $91.00\left(9.500, \mathrm{M}^{+}-\mathrm{C}_{8} \mathrm{H}_{17}\right)$.

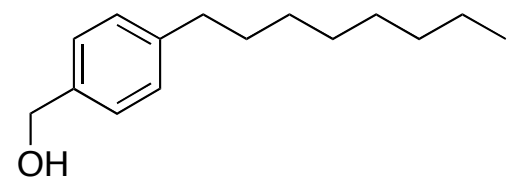

(4-octylphenyl)methanol (3h) (Table 1, entry 8) [CAS: 40016-25-9]. The general procedure was followed with 4-iodo(phenyl)methanol (234 $\mathrm{mg}, 1.00 \mathrm{mmol}, 1.00$ equiv) and 1-iodooctane $\left(181 \mu \mathrm{L}, 1.00 \mathrm{mmol}, 1.00\right.$ equiv) at $80^{\circ} \mathrm{C}$ for $15 \mathrm{~h}$. The product was isolated by chromatography (50:50 pentane:ether, first run and 80:20 hexanes:EtOAc, second run) as colorless oil. First run $165 \mathrm{mg}(75 \%)$. Second run $179 \mathrm{mg}(81 \%)$.

${ }^{1} \mathrm{H}$ NMR $\left(500 \mathrm{MHz}, \mathrm{CDCl}_{3}\right) \delta 7.32(\mathrm{~d}, J=8.0 \mathrm{~Hz}, 2 \mathrm{H}), 7.23(\mathrm{~d}, J=8.0 \mathrm{~Hz}, 2 \mathrm{H}), 4.68(\mathrm{~s}$, $2 \mathrm{H}), 2.67$ (t, $J=7.6 \mathrm{~Hz}, 2 \mathrm{H}), 2.15$ (br s, $1 \mathrm{H}), 1.69-1.66(\mathrm{~m}, 2 \mathrm{H}), 1.38-1.34(\mathrm{~m}, 10 \mathrm{H}), 0.96(\mathrm{t}, J=$ $5.0 \mathrm{~Hz}, 3 \mathrm{H})$.

${ }^{13} \mathrm{C}$ NMR $\left(125 \mathrm{MHz}, \mathrm{CDCl}_{3}\right) \delta$ 142.6, 138.2, 128.7, 127.2, 65.3, 35.8, 32.0, 31.7, 29.6, 29.44, 29.39, 22.8, 14.2.

GC-MS $m / z$ (\% relative intensity, ion): $220.05\left(19.70, \mathrm{M}^{+}\right), 121.05\left(70.50, \mathrm{M}^{+}-\mathrm{C}_{7} \mathrm{H}_{15}\right)$, $107.00\left(100.0, \mathrm{M}^{+}-\mathrm{C}_{8} \mathrm{H}_{17}\right), 91.00\left(41.6, \mathrm{M}^{+}-\mathrm{C}_{8} \mathrm{H}_{18} \mathrm{O}\right)$.

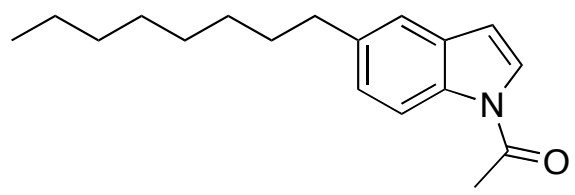

1-(5-octyl-1 $H$-indol-1-yl)ethanone (3i) (Table 1, entry 9). The reaction was run according to the general procedure except 4,4'-di-tert-butyl-2,2'-dipyridyl (26.8 mg, $0.100 \mathrm{mmol}, 0.100$ equiv) was used and no 1,2-bis(diphenylphosphino)benzene was used. Accordingly, $N$-acetyl-5bromoindole ( $239 \mathrm{mg}, 1.00 \mathrm{mmol}, 1.00$ equiv), and 1-iodooctane $(181 \mu \mathrm{L}, 1.00 \mathrm{mmol}, 1.00$ equiv) were heated at $60{ }^{\circ} \mathrm{C}$ for $14 \mathrm{~h}$. The product was isolated by chromatography (hexanes:EtOAc 85:15) as yellow oil. First run $227 \mathrm{mg}(84 \%)$. Second run $189 \mathrm{mg}(70 \%)$.

${ }^{1} \mathrm{H}$ NMR $\left(500 \mathrm{MHz}, \mathrm{CDCl}_{3}\right) \delta 8.32$ (s, br., $\left.1 \mathrm{H}\right), 7.39-7.36(\mathrm{~m}, 2 \mathrm{H}), 7.18(\mathrm{dd}, 1 \mathrm{H}, J=8.5$ $\mathrm{Hz}$ and $1.6 \mathrm{~Hz}), 6.59(\mathrm{~d}, J=3.7 \mathrm{~Hz}, 1 \mathrm{H}), 2.70(\mathrm{t}, J=7.6 \mathrm{~Hz}, 2 \mathrm{H}), 2.62(\mathrm{~s}, 3 \mathrm{H}), 1.68-1.62(\mathrm{~m}$, $2 \mathrm{H}), 1.34-1.27(\mathrm{~m}, 10 \mathrm{H}), 0.88(\mathrm{t}, J=7.1 \mathrm{~Hz}, 3 \mathrm{H})$.

${ }^{13} \mathrm{C}$ NMR $\left(125 \mathrm{MHz}, \mathrm{CDCl}_{3}\right) \delta 168.5,138.6,134.0,130.7,126.0,125.3,120.3,116.3$, $109.2,36.0,32.1,32.0,29.6,29.44,29.40,24.0,22.8,14.2$.

GC-MS $m / z$ (\% relative intensity, ion): $271.10\left(14.50, \mathrm{M}^{+}\right), 229.10\left(14.10, \mathrm{M}^{+}-\mathrm{C}_{2} \mathrm{H}_{2} \mathrm{O}\right)$, $172.15\left(10.50, \mathrm{M}^{+}-\mathrm{C}_{7} \mathrm{H}_{17}\right), 130.10\left(100.0, \mathrm{M}^{+}-\mathrm{C}_{9} \mathrm{H}_{17} \mathrm{O}\right)$. 
<smiles>CCOC(=O)CCCc1ccccc1</smiles>

Ethyl 4-phenylbutanoate (3j) (Table 1, entry 10) [CAS: 10031-93-3]. ${ }^{10}$ The general procedure was followed with iodobenzene ( $112 \mu \mathrm{L}, 1.00 \mathrm{mmol}, 1.00$ equiv) and ethyl-4-bromobutyrate $\left(145 \mu \mathrm{L}, 1.00 \mathrm{mmol}, 1.00\right.$ equiv) at $80^{\circ} \mathrm{C}$ for $7 \mathrm{~h}$. The product was isolated by chromatography (80:20 pentane:ether, first run and 95:5 pentane:ether, second run) as colorless oil. First run 162 $\mathrm{mg}(84 \%)$. Second run $135 \mathrm{mg}(70 \%)$.

${ }^{1} \mathrm{H}$ NMR $\left(500 \mathrm{MHz}, \mathrm{CDCl}_{3}\right) \delta$ 7.31-7.27 (m, 2H), 7.21-7.18 (m, 3H), 4.13 (q, 2H, $J=$ $7.1 \mathrm{~Hz}), 2.65$ (t, $J=7.4 \mathrm{~Hz}, 2 \mathrm{H}), 2.32$ (t, $J=7.4 \mathrm{~Hz}, 2 \mathrm{H}), 1.99-1.92(\mathrm{~m}, 2 \mathrm{H}), 1.26$ (t, $J=7.1 \mathrm{~Hz}$, $3 \mathrm{H})$.

${ }^{13} \mathrm{C}$ NMR $\left(125 \mathrm{MHz}, \mathrm{CDCl}_{3}\right) \delta 173.6,141.6,128.6,128.5,126.1,60.4,35.3,33.8,26.7$, 14.4 .

GC-MS $m / z$ (\% relative intensity, ion): $192.10\left(21.20, \mathrm{M}^{+}\right), 147.10\left(42.50, \mathrm{M}^{+}-\mathrm{C}_{2} \mathrm{H}_{5} \mathrm{O}\right)$, $104.00\left(90.00, \mathrm{M}^{+}-\mathrm{C}_{4} \mathrm{H}_{8} \mathrm{O}_{2}\right), 91.00\left(100.0, \mathrm{M}^{+}-\mathrm{C}_{5} \mathrm{H}_{9} \mathrm{O}_{2}\right)$.<smiles>CC(C)(C)OC(=O)NCCCc1ccccc1</smiles>

tert- butyl 3-phenylpropylcarbamate (3k) (Table 1, entry 11) [CAS: 147410-39-7]. The general procedure was followed with iodobenzene $(112 \mu \mathrm{L}, 1.00 \mathrm{mmol}, 1.00$ equiv) and 3bromopropyl-carbamic acid tert-butyl ester $\left(238 \mathrm{mg}, 1.00 \mathrm{mmol}, 1.00\right.$ equiv) at $60{ }^{\circ} \mathrm{C}$ for $16 \mathrm{~h}$. The product was isolated by chromatography (hexanes:EtOAc 1-10\% EtOAc) as yellow oil. First run $193 \mathrm{mg}$ (83\%). Second run $206 \mathrm{mg}(89 \%)$.

${ }^{1} \mathrm{H}$ NMR (500 MHz, $\left.\mathrm{CDCl}_{3}\right) \delta$ 7.36-7.33 (m, 2H), 7.26-7.23 (m, 3H), 4.86 (s, br., 1H), 3.22-3.21 (m, br., $2 \mathrm{H}), 2.70(\mathrm{t}, J=7.6 \mathrm{~Hz}, 2 \mathrm{H}), 1.90-1.84(\mathrm{~m}, 2 \mathrm{H}), 1.53(\mathrm{~s}, 9 \mathrm{H})$.

${ }^{13} \mathrm{C}$ NMR $\left(125 \mathrm{MHz}, \mathrm{CDCl}_{3}\right) \delta 156.0,141.6,128.40,128.36,125.9,19.0,40.2,33.1$, 31.7, 28.4.

GC-MS $m / z$ (\% relative intensity, ion): $179.10\left(23.30, \mathrm{M}^{+}-\mathrm{C}_{4} \mathrm{H}_{8+}\right), 118.05\left(100.0, \mathrm{M}^{+}-\right.$ $\left.\mathrm{C}_{5} \mathrm{H}_{11} \mathrm{NO}_{2}\right), 91.00\left(15.70, \mathrm{M}^{+}-\mathrm{C}_{7} \mathrm{H}_{14} \mathrm{NO}_{2}\right), 57.00\left(77.50, \mathrm{M}^{+}-\mathrm{C}_{11} \mathrm{H}_{14} \mathrm{O}_{2}\right)$.<smiles>O=C(NCCCc1ccccc1)OCc1ccccc1</smiles>

Benzyl 3-phenylpropylcarbamate (3I) (Table 1, entry 12) [CAS: 302569-84-2]. ${ }^{11}$ The general procedure was followed with iodobenzene $(112 \mu \mathrm{L}, 1.00 \mathrm{mmol}, 1.00$ equiv) and 3-bromopropylcarbamic acid benzyl ester $\left(272 \mathrm{mg}, 1.00 \mathrm{mmol}, 1.00\right.$ equiv) at $60^{\circ} \mathrm{C}$ for $14 \mathrm{~h}$. The product was isolated by chromatography (hexanes:EtOAc $3-35 \%$ EtOAc) as yellow oil. First run $158 \mathrm{mg}$ (59\%). Second run $191 \mathrm{mg}(71 \%)$.

${ }^{1} \mathrm{H}$ NMR $\left(400 \mathrm{MHz}, \mathrm{CDCl}_{3}\right) \delta$ 7.38-7.18 (m, $\left.10 \mathrm{H}\right), 5.13$ (s, 2H), 4.90 (s, br., 1H), 3.28$3.20(\mathrm{~m}, 2 \mathrm{H}), 2.66(\mathrm{t}, J=7.7 \mathrm{~Hz}, 2 \mathrm{H}), 1.89-1.82(\mathrm{~m}, 2 \mathrm{H})$.

${ }^{13} \mathrm{C}$ NMR (101 MHz, $\left.\mathrm{CDCl}_{3}\right) \delta 156.5,141.4,136.6,128.47,128.45,128.4,128.3,128.0$, 125.9, 66.5, 40.6, 33.0, 31.5.

GC-MS $m / z$ (\% relative intensity, ion): $269.10\left(0.800, \mathrm{M}^{+}\right), 178.15\left(30.20, \mathrm{M}^{+}-\mathrm{C}_{7} \mathrm{H}_{7}\right)$, $91.00\left(100.0, \mathrm{M}^{+}-\mathrm{C}_{10} \mathrm{H}_{12} \mathrm{NO}_{2}\right)$. 


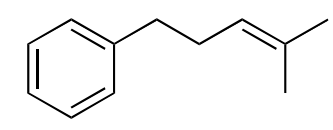

1-(4-methylpent-3-enyl)benzene (3m) (Table 1, entry 13)[CAS: 33501-90-5]. ${ }^{12}$ The general procedure was followed with iodobenzene $(112 \mu \mathrm{L}, 1.00 \mathrm{mmol}, 1.00$ equiv) and 5-bromo-2methyl-2-pentene (133 $\mu \mathrm{L}, 1.00 \mathrm{mmol}, 1.00$ equiv) at $60{ }^{\circ} \mathrm{C}$ for $37 \mathrm{~h}$. The product was isolated by chromatography (hexanes) as yellow oil. First run $111 \mathrm{mg}$ (69\%). Second run $93.1 \mathrm{mg}(58 \%)$.

${ }^{1} \mathrm{H}$ NMR $\left(400 \mathrm{MHz}, \mathrm{CDCl}_{3}\right) \delta$ 7.40-7.26 (m, 5H), 5.32-5.28 (m, 1H), $2.75(\mathrm{t}, J=7.5 \mathrm{~Hz}$, 2H), $2.43(\mathrm{q}, J=7.7 \mathrm{~Hz}, 2 \mathrm{H}), 1.81(\mathrm{~s}, 3 \mathrm{H}), 1.68(\mathrm{~s}, 3 \mathrm{H})$.

${ }^{13} \mathrm{C}$ NMR $\left(101 \mathrm{MHz}, \mathrm{CDCl}_{3}\right) \delta 142.5,132.2,128.6,128.3,125.8,123.9,36.3,30.2,25.8$, 17.8 .

GC-MS $m / z$ (\% relative intensity, ion): $160.20\left(27.30, \mathrm{M}^{+}\right), 91.00\left(44.40, \mathrm{M}^{+}-\mathrm{C}_{5} \mathrm{H}_{9}\right)$, $69.05\left(100.0, \mathrm{M}^{+}-\mathrm{C}_{7} \mathrm{H}_{7}\right)$.<smiles>CCC(CC)Cc1ccccc1</smiles>

2-ethylhexylbenzene (3n) (Table 1, entry 14). ${ }^{13}$ The general procedure was followed with iodobenzene $(112 \mu \mathrm{L}, 1.00 \mathrm{mmol}, 1.00$ equiv) and 2-ethylhexylbromide (178 $\mu \mathrm{L}, 1.00 \mathrm{mmol}$, 1.00 equiv) at $80^{\circ} \mathrm{C}$ for $7 \mathrm{~h}$. The product was isolated by preparative TLC (hexanes, $\mathrm{R}_{\mathrm{f}}=0.8$ ) as a yellow oil. First run $134 \mathrm{mg}(71 \%)$. Second run $149 \mathrm{mg}(78 \%)$.

${ }^{1} \mathrm{H}$ NMR $\left(400 \mathrm{MHz}, \mathrm{CDCl}_{3}\right) \delta$ 7.39-7.36 (m, 2H), 7.29-7.26 (m, 3H), 2.71-2.62 (m, 2H), 1.75-1.66 (m, 1H), 1.45-1.372 (m, 8H), 1.03-0.99 (m, 6H)

${ }^{13} \mathrm{C} \mathrm{NMR}\left(101 \mathrm{MHz}, \mathrm{CDCl}_{3}\right) \delta$ 142.0, 129.3, 128.2, 125.6, 41.3, 40.3, 32.5, 29.0, 25.6, $23.2,14.3,10.9$.

GC-MS $m / z$ (\% relative intensity, ion): $190.20\left(15.70, \mathrm{M}^{+}\right), 98.00\left(15.90, \mathrm{M}^{+}-\mathrm{C}_{7} \mathrm{H}_{14}\right)$, $91.01\left(55.60, \mathrm{M}^{+}-\mathrm{C}_{7} \mathrm{H}_{7}\right), 57.02\left(100.0, \mathrm{M}^{+}-\mathrm{C}_{4} \mathrm{H}_{9}\right)$<smiles>CC(C)(C)Cc1ccccc1</smiles>

neo-pentylbenzene (3o) (Table 1, entry 15) [CAS: 1007-26-7]. ${ }^{14}$ The general procedure was followed with iodobenzene $(112 \mu \mathrm{L}, 1.00 \mathrm{mmol}, 1.00$ equiv) and neo-pentyliodide $(159 \mu \mathrm{L}, 1.00$ mmol, 1.00 equiv) at $80^{\circ} \mathrm{C}$ for $21 \mathrm{~h}$. The product was isolated by chromatography (hexanes) as colorless oil. First run $54 \mathrm{mg}(36 \%)$. Second run $60 \mathrm{mg}(41 \%)$.

${ }^{1} \mathrm{H}$ NMR $\left(400 \mathrm{MHz}, \mathrm{CDCl}_{3}\right) \delta$ 7.33-7.17 (m, 5H), 2.55 (s, 2H), 0.97 (s, 9H).

${ }^{13} \mathrm{C} \mathrm{NMR}\left(101 \mathrm{MHz}, \mathrm{CDCl}_{3}\right) \delta$ 139.9, 130.6, 127.7, 125.9, 50.4, 31.9, 29.6.

GC-MS $m / z$ (\% relative intensity, ion): $148.20\left(21.20, \mathrm{M}^{+}\right), 133.15\left(12.10, \mathrm{M}^{+}-\mathrm{CH}_{3}\right)$, $92.00\left(100.0, \mathrm{M}^{+}-\mathrm{C}_{4} \mathrm{H}_{8}\right), 57.00\left(94.40, \mathrm{M}^{+}-\mathrm{C}_{7} \mathrm{H}_{7}\right)$.<smiles>CCCCCC(C)c1ccccc1</smiles>

1-(heptan-2-yl)benzene (3p) (Table 1, entry 16) [CAS: 2132-84-5]. ${ }^{15}$ The general procedure was followed with iodobenzene $(112 \mu \mathrm{L}, 1.00 \mathrm{mmol}, 1.00$ equiv) and 2-bromoheptane $(188 \mu \mathrm{L}$, $1.20 \mathrm{mmol}, 1.20$ equiv) at $60{ }^{\circ} \mathrm{C}$ for $26 \mathrm{~h}$. The product was isolated by chromatography (hexanes) 
as colorless oil. First run $152 \mathrm{mg}$ (86\%, isolated as a 95:5 mixture with (heptan-3-yl)benzene). Second run $159 \mathrm{mg}$ (90\% yield, isolated as a 95:5 mixture with (heptan-3-yl)benzene). Reaction selectivity determined to be 89:7:4 3p:branched isomer:linear isomer by GC analysis of the crude reaction mixture.

${ }^{1} \mathrm{H}$ NMR $\left(400 \mathrm{MHz}, \mathrm{CDCl}_{3}\right) \delta$ 7.42-7.26 (m, 5H), 2.86-2.77 (m, 1H), 1.74-1.69 (m, 2H), $1.39-1.37(\mathrm{~m}, 9 \mathrm{H}), 1.00(\mathrm{t}, J=6.7 \mathrm{~Hz}, 3 \mathrm{H})$.

${ }^{13} \mathrm{C}$ NMR $\left(101 \mathrm{MHz}, \mathrm{CDCl}_{3}\right) \delta 148.1,128.4,127.1,125.9,40.2,38.6,32.2,27.6,22.8$, $22.5,14.2$.

GC-MS $m / z$ (\% relative intensity, ion): $176.20\left(8.8, \mathrm{M}^{+}\right), 105.00\left(100.0, \mathrm{M}^{+}-\mathrm{C}_{5} \mathrm{H}_{11}\right)$, $91.00\left(9.9, \mathrm{M}^{+}-\mathrm{C}_{6} \mathrm{H}_{14}\right)$.

${ }^{1}$ Ward, L. G. L.; Pipal, J. R. Inorg. Synth. 1971, 13, 154.

${ }^{2}$ Georgiades, S. N.; Clardy, J. Org. Lett. 2005, 7, 4091.

${ }^{3}$ Robarge, M. J.; Husbands, S. M.; Kieltyka, A.; Brodbeck, R.; Thurkauf, A.; Newman, A. H. J. Med. Chem. 2001, 44, 3175.

${ }^{4}$ Koolmeister, T.; Södergren, M.; Scobie, M. Tetrahedron Lett. 2002, 43, 5965.

${ }^{5}$ Phipps, R. J.; Brimster, N. P.; Gaunt, M. J. J. Am. Chem. Soc. 2008, 130, 8172.

${ }^{6}$ Schreiber, S.T.; Shair, M. D.; Tan, D.S.; Foley, M. A.; Stockwell, B. R. Synthesis of Combinatorial Libraries of Compounds Reminiscent of Natural Products. U.S. Patent 6,448,443, September 10, 2002.

${ }^{6}$ Merrill, R. E.; Negishi, E. J. Org. Chem. 1974, 39, 3452.

${ }^{7}$ Soulie, C. Tetrahedron. 2001, 57, 1035.

${ }^{8}$ Kondolff, I.; Doucet, H.; Santelli, M. Tetrahedron. 2004, 60, 3813.

${ }^{9}$ Huo, S. Org. Lett. 2003, 5, 423.

${ }^{10}$ Kurono, Nobuhito.; Sugita, K.; Takasugi, S.; Tokuda, M. Tetrahedron. 1999, 55, 6097.

${ }^{11}$ Yang, B-L.; Tian, S-K. Eur. J. Org. Chem. 2007, 28, 4646.

${ }^{12}$ Charette, A. B.; Molinaro, C.; Brochu, C. J. Am. Chem. Soc. 2001, 123, 12160.

${ }^{13}$ (a) Fessard, T. C.; Motoyoshi, H.; Carreira, E. M. Angew. Chem. Int. Ed. 2007, 46, 2078; (b) Toussaint, H. J. Am .Chem. Soc. 1940, 62, 1145.

${ }^{14}$ Martin, R.; Fürstner, A. Angew. Chem. Int. Ed. 2004, 43, 3955.

${ }^{15}$ Hobbs, C.; Hammann, W. J. Org. Chem. 1970, 35 4188-4191 


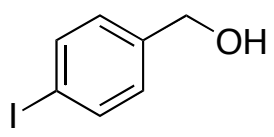




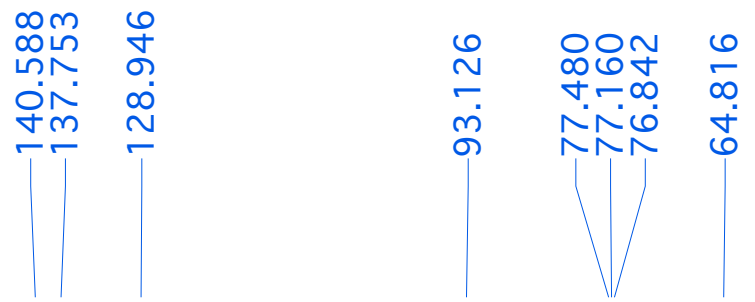
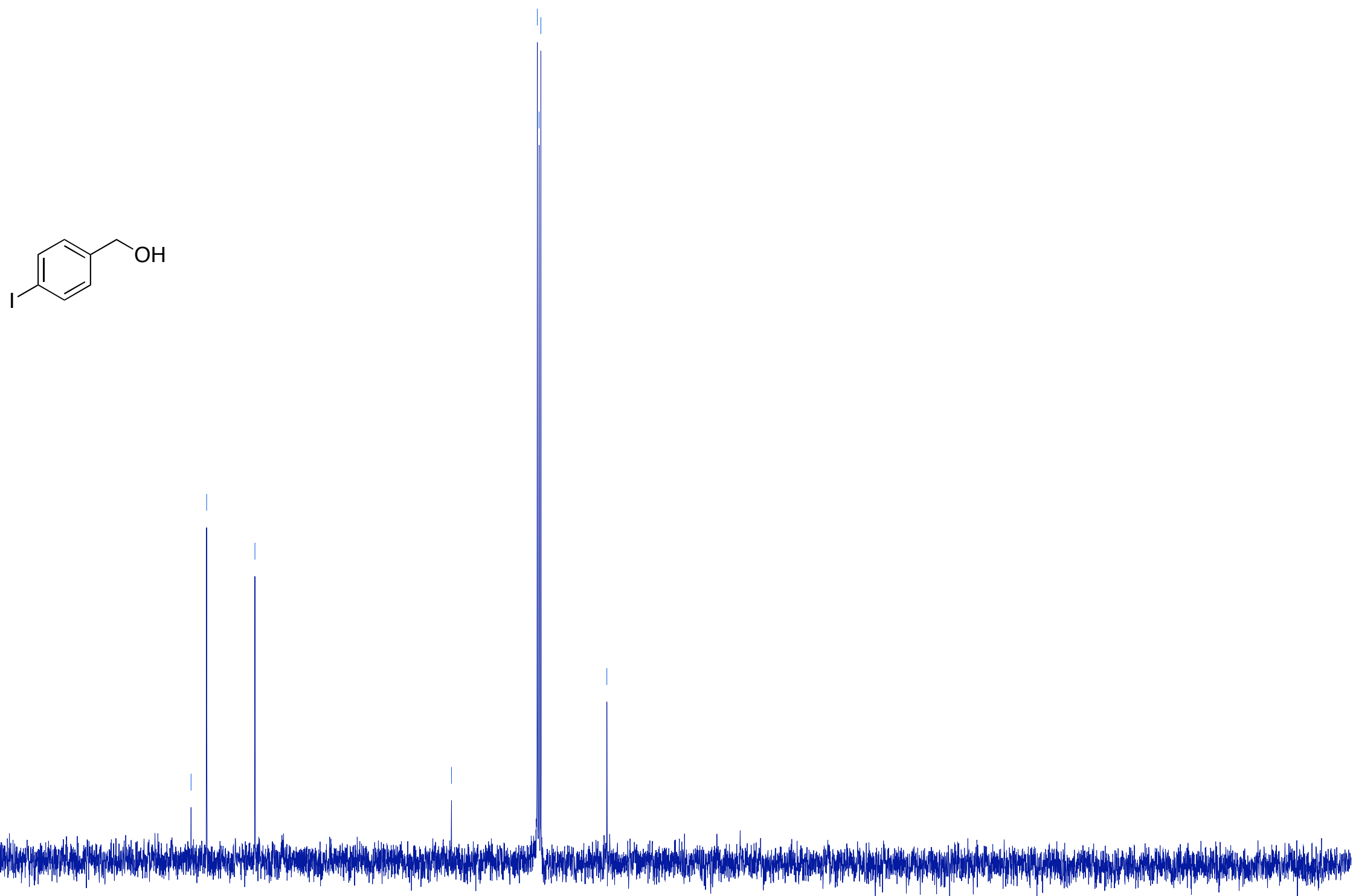


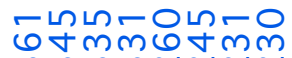

mmmMNNNN

NNNNN

$+\infty m$

806

inin

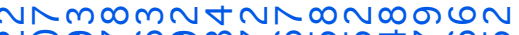

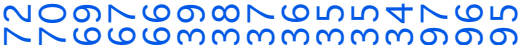

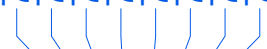

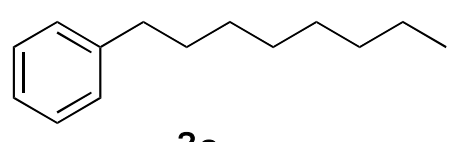

$3 a$

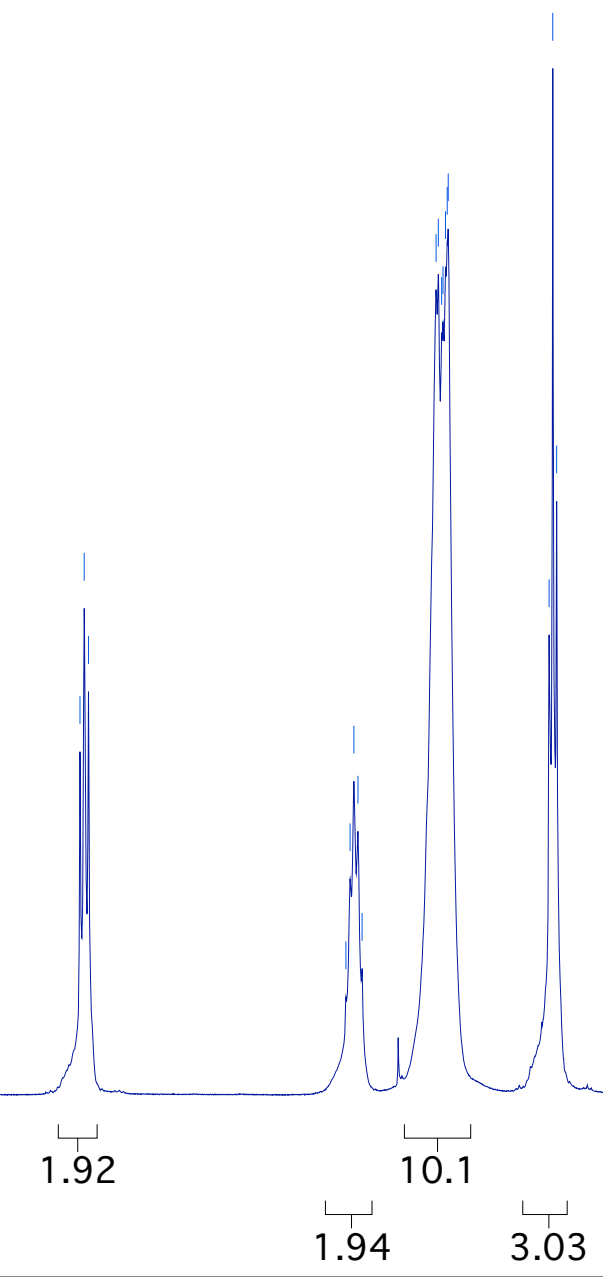




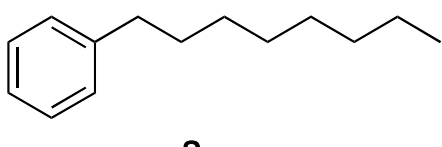




\section{S20}

느뉴

Ninं

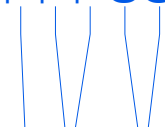

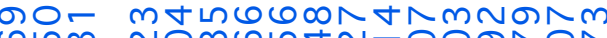
作 NNN

$3 b$

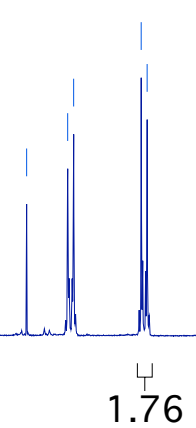

1.77
2.72
4

\section{4}

1.95

3 


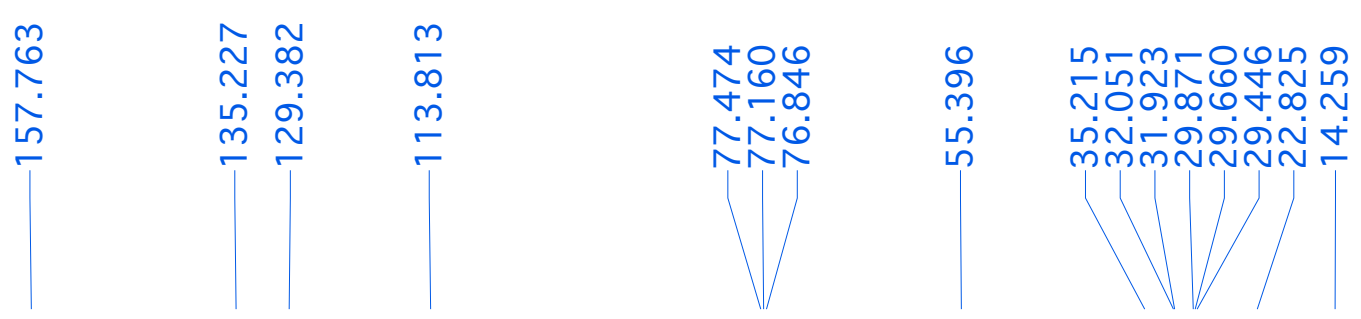

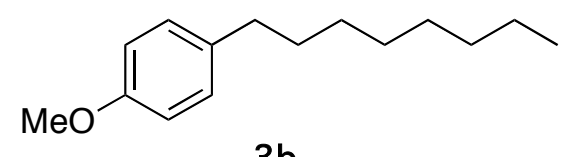

$3 b$

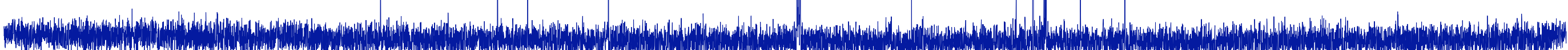
M.m.

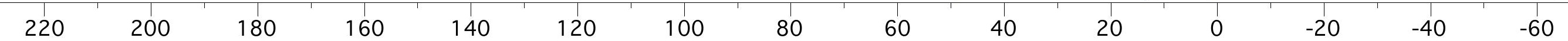


$\forall \infty$

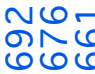

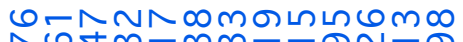

นกMNN

ง.06

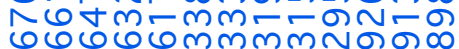

Nก

-ー- -

舟

$3 c$

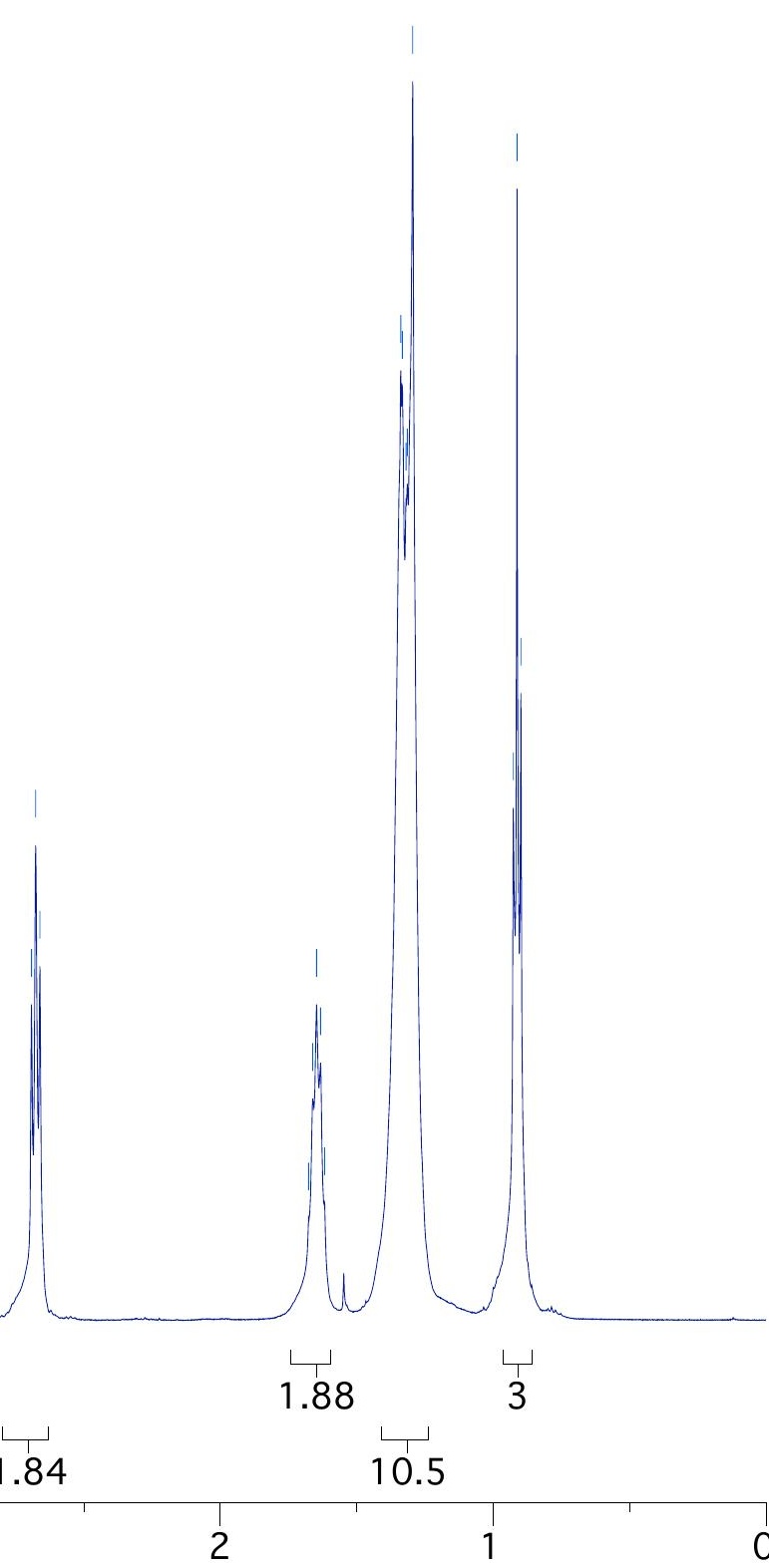




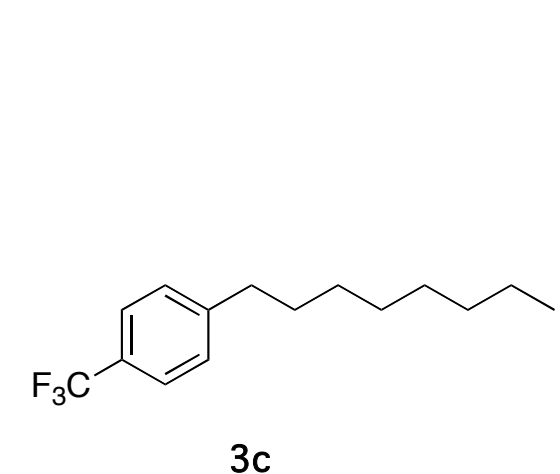

$3 c$
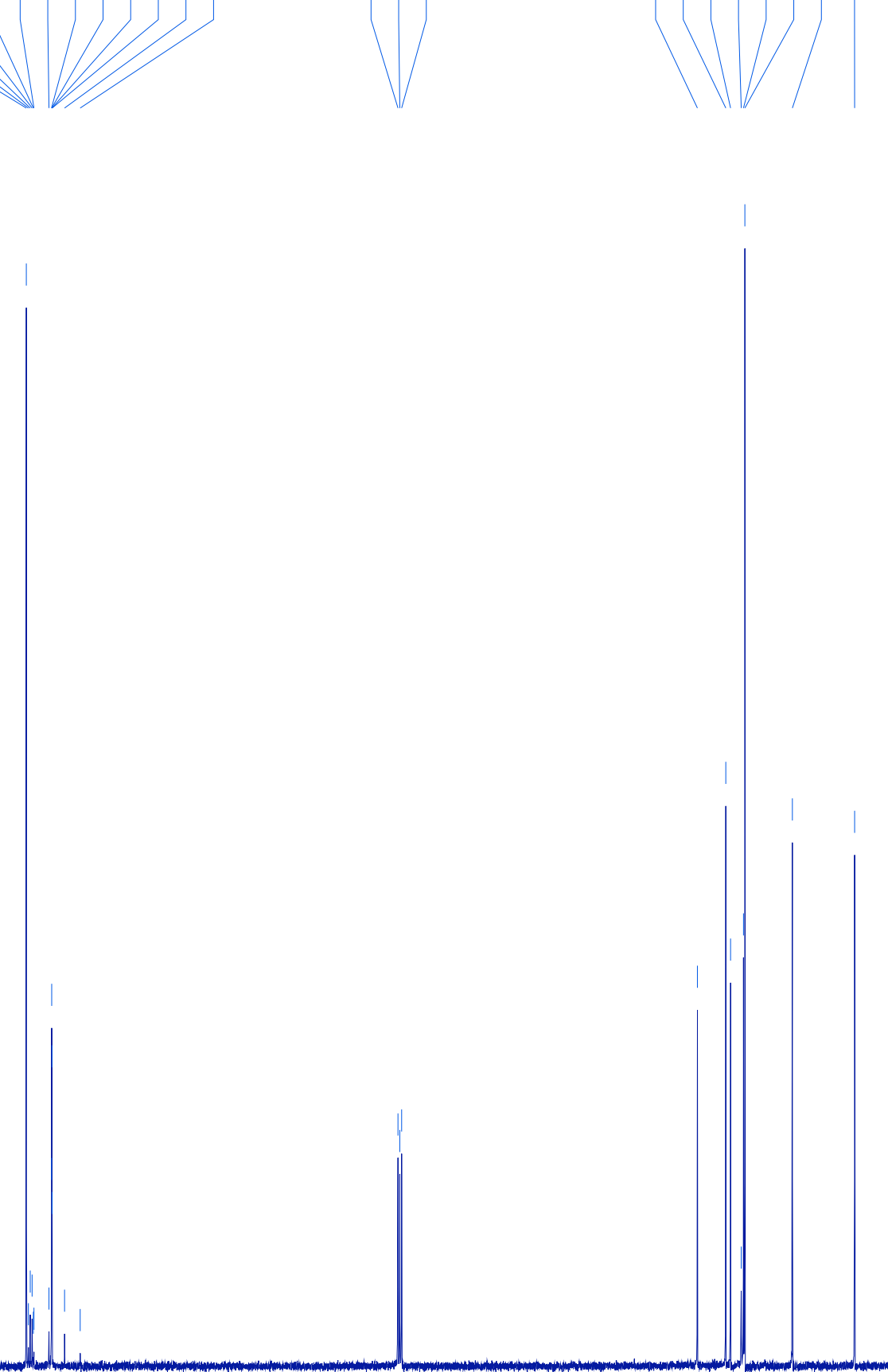


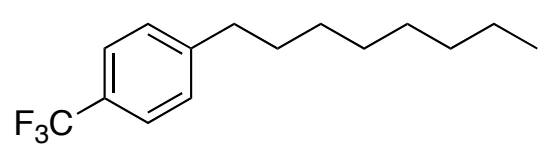

$3 c$ 
mNonNL-mGo

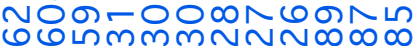

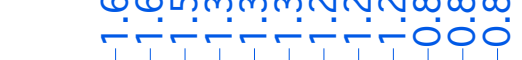

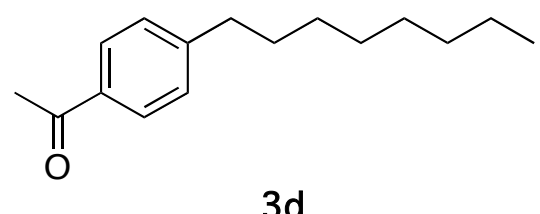

$3 d$

\begin{tabular}{|c|c|c|}
\hline 3.03 & \multicolumn{2}{|c|}{10.6} \\
\hline 2.03 & 2.18 & 3.28 \\
\hline
\end{tabular}



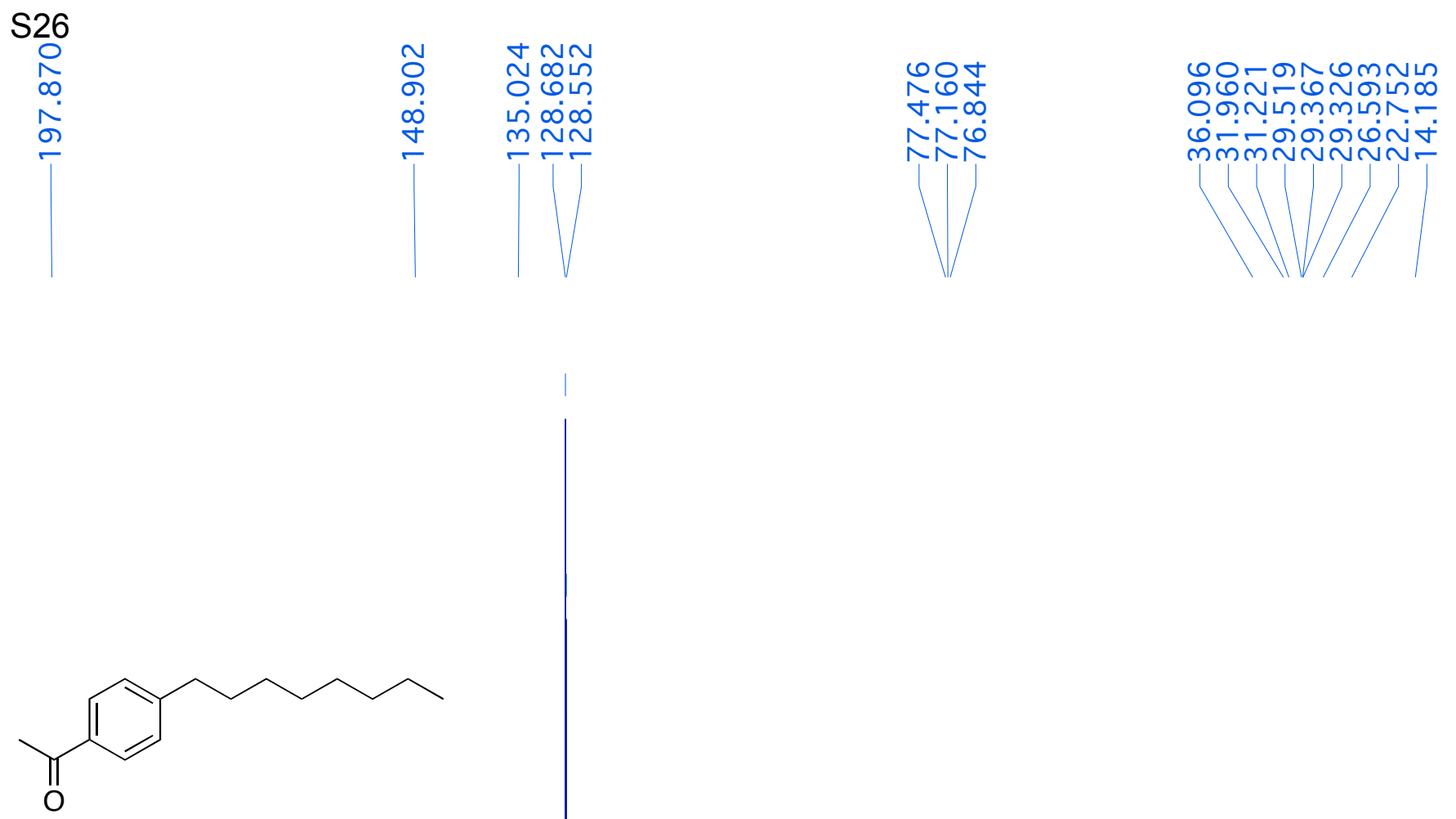

$3 d$

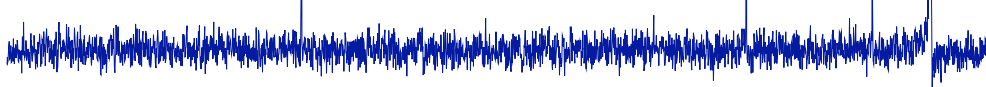

ดยก-

- -

MmMNNNNN- 
เับกำ

แกบกำ

NNNN

시요

ช6ำ

กัก

\&UNDOMNDNtm-t ชช ชิ -ா-г-г-гі0

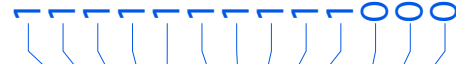

V<smiles>CCCCCCCCc1ccc(C#N)cc1</smiles>

$3 e$
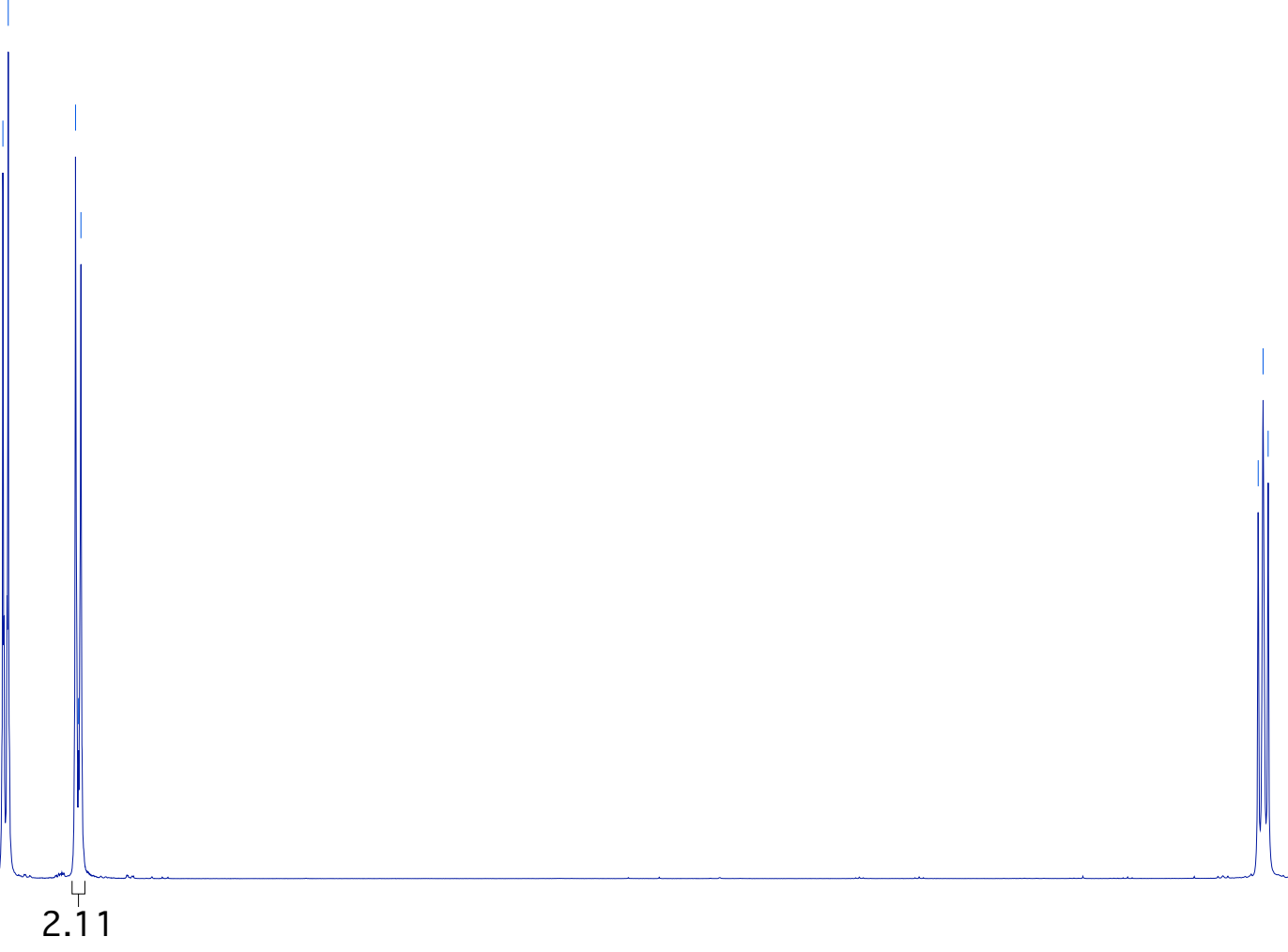

2.17 2.28

2 

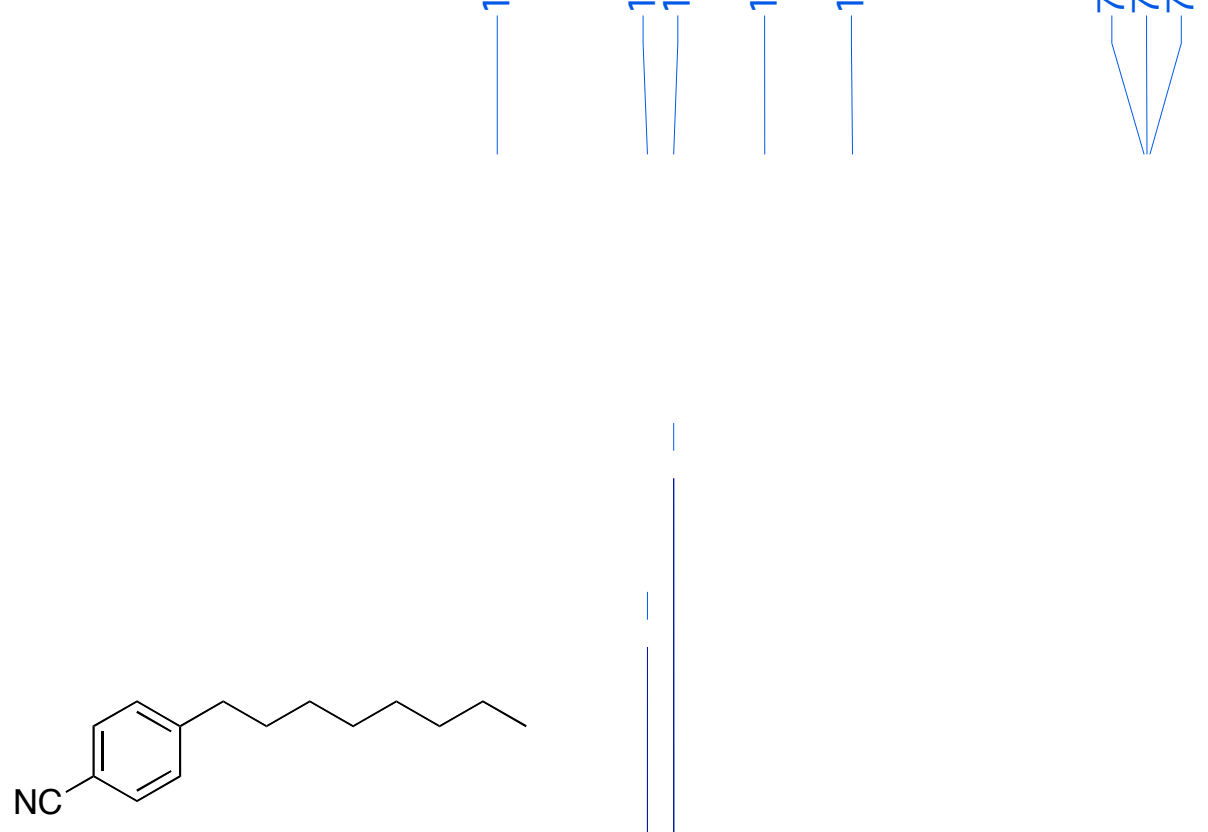

$3 e$ 
N

N …<smiles>CCCCCCCCc1ccc(B2OC(C)(C)C(C)(C)O2)cc1</smiles>

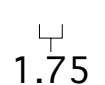

1.77

8

1.83 


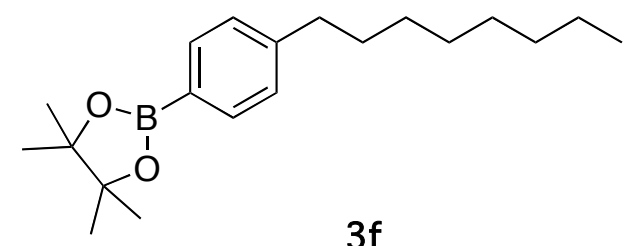

$3 f$ 

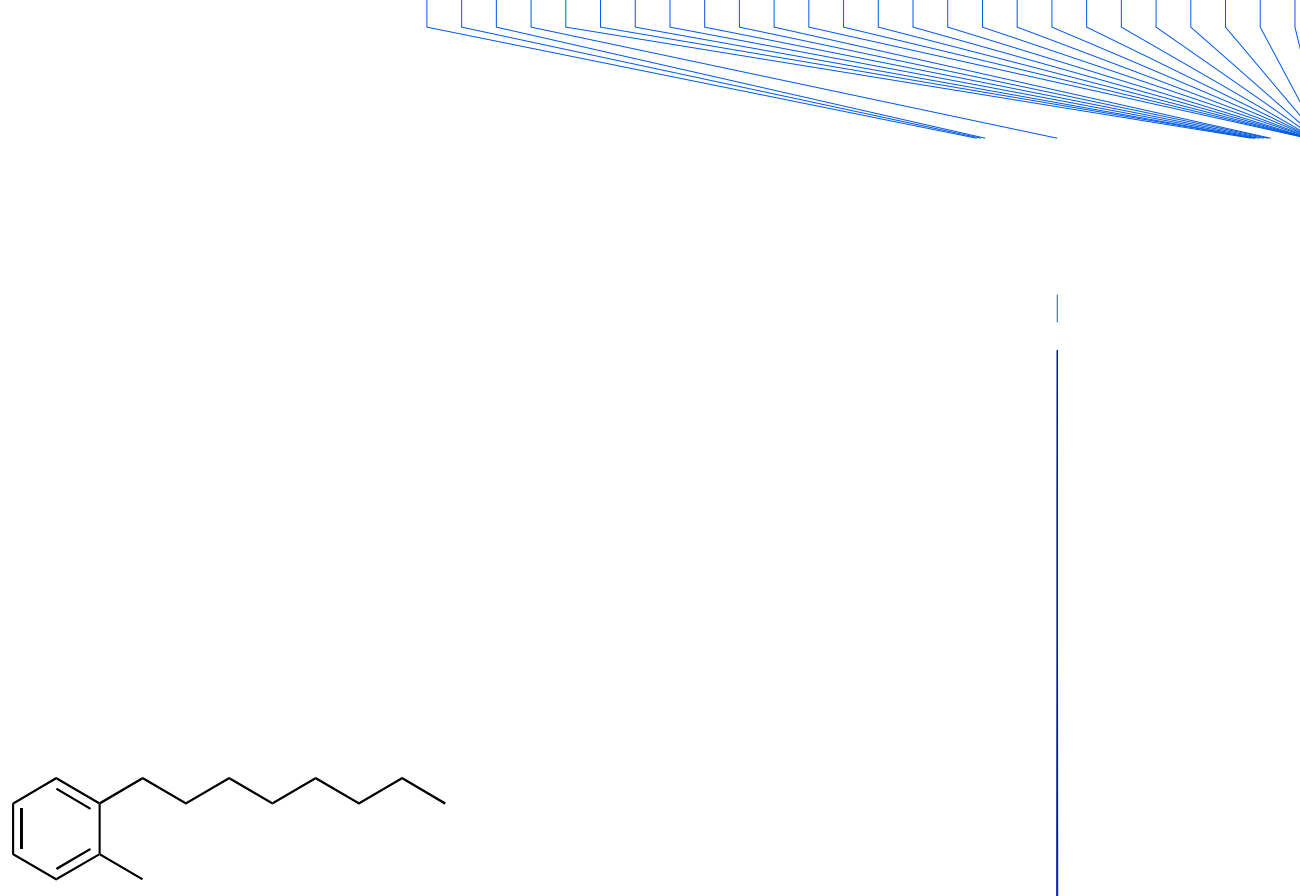

$3 g$

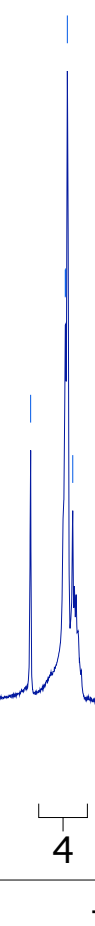




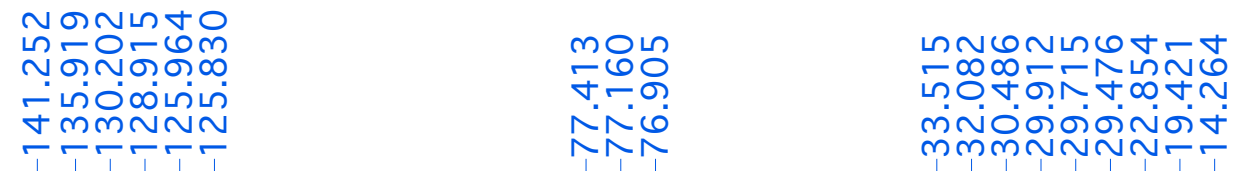

$3 g$

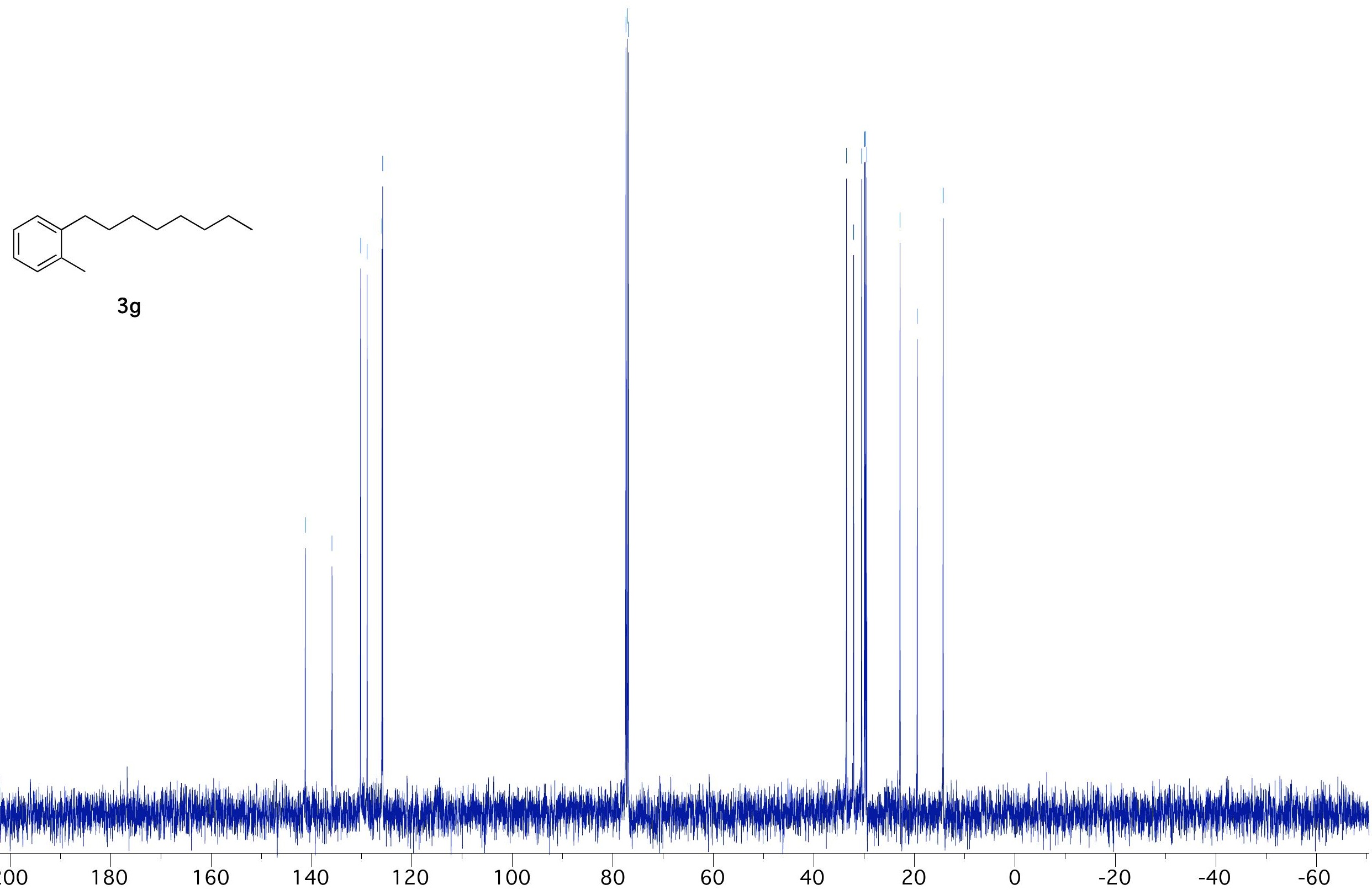


ก

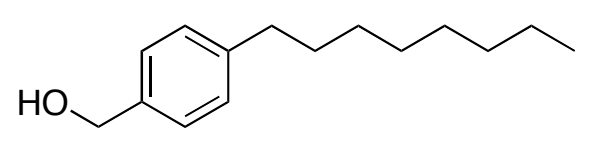

$3 h$

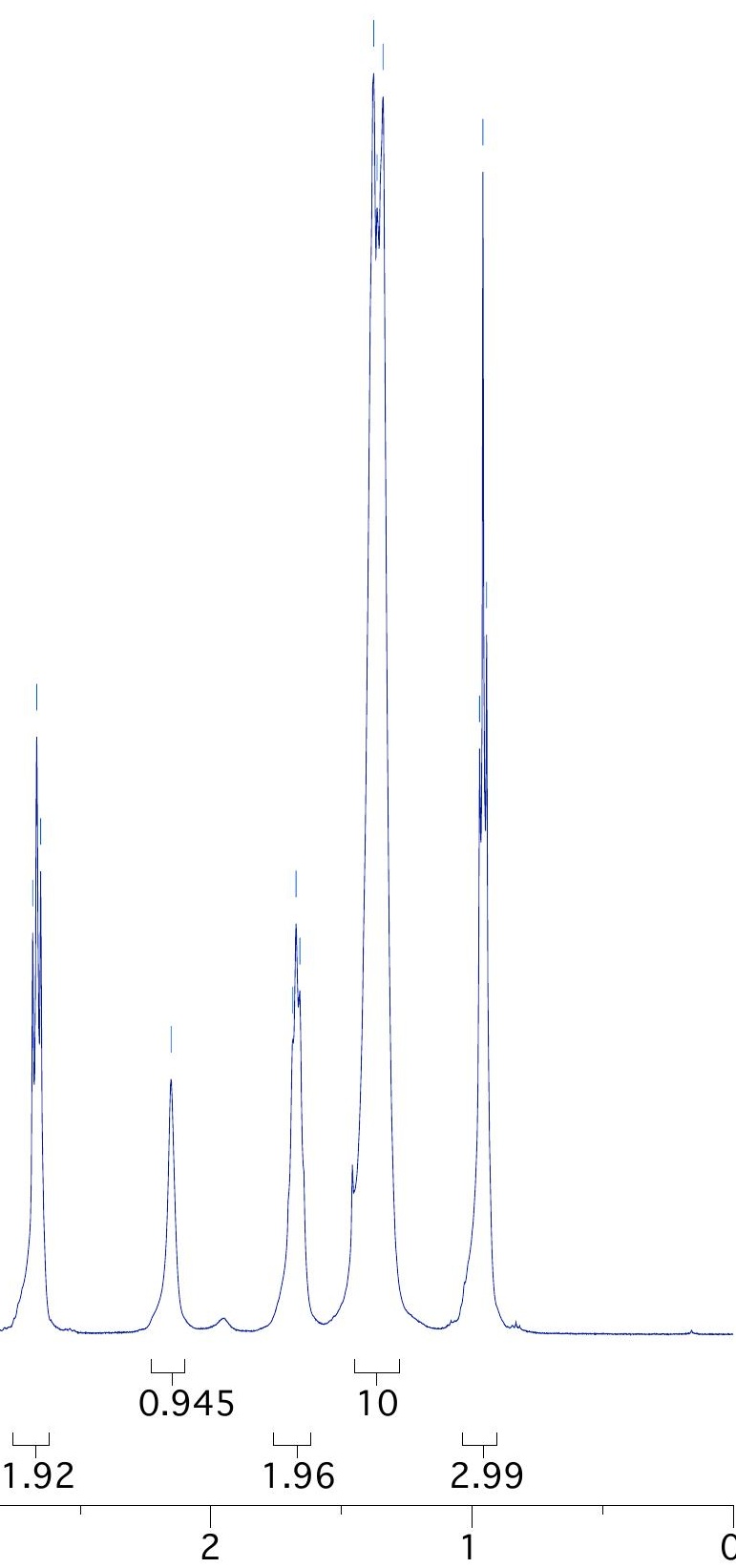


S34
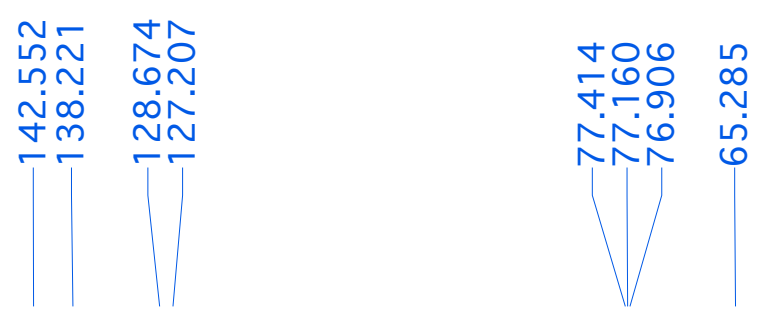

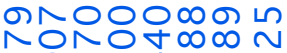
No6.tmN N

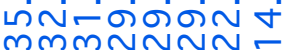<smiles>CCCCCCCCc1ccc(CO)cc1</smiles>

$3 \mathrm{~h}$

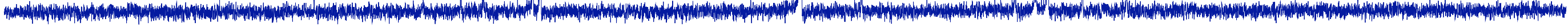




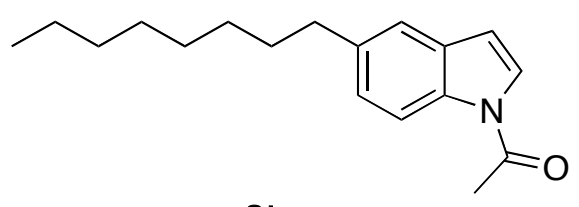

$3 i$

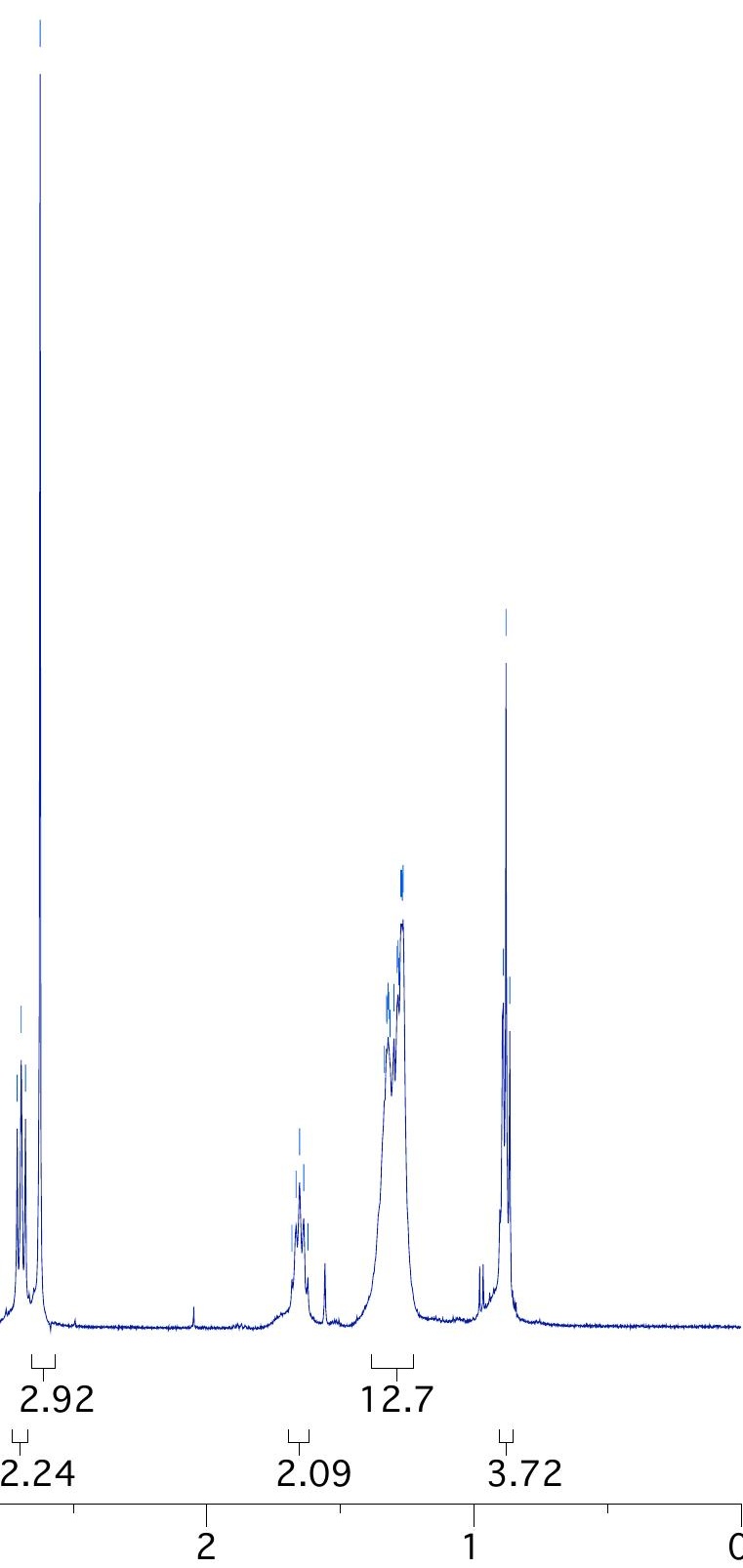


S36

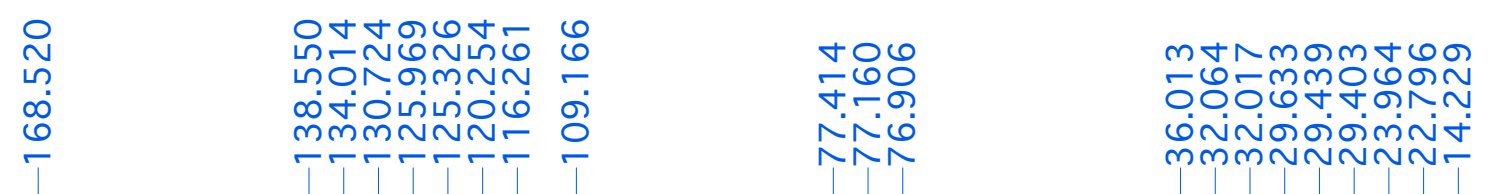

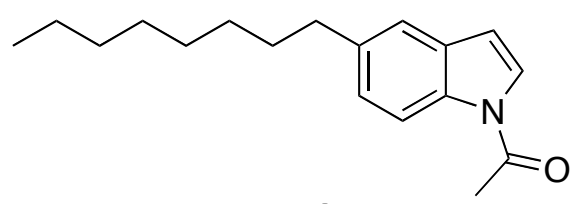

$3 i$

NN
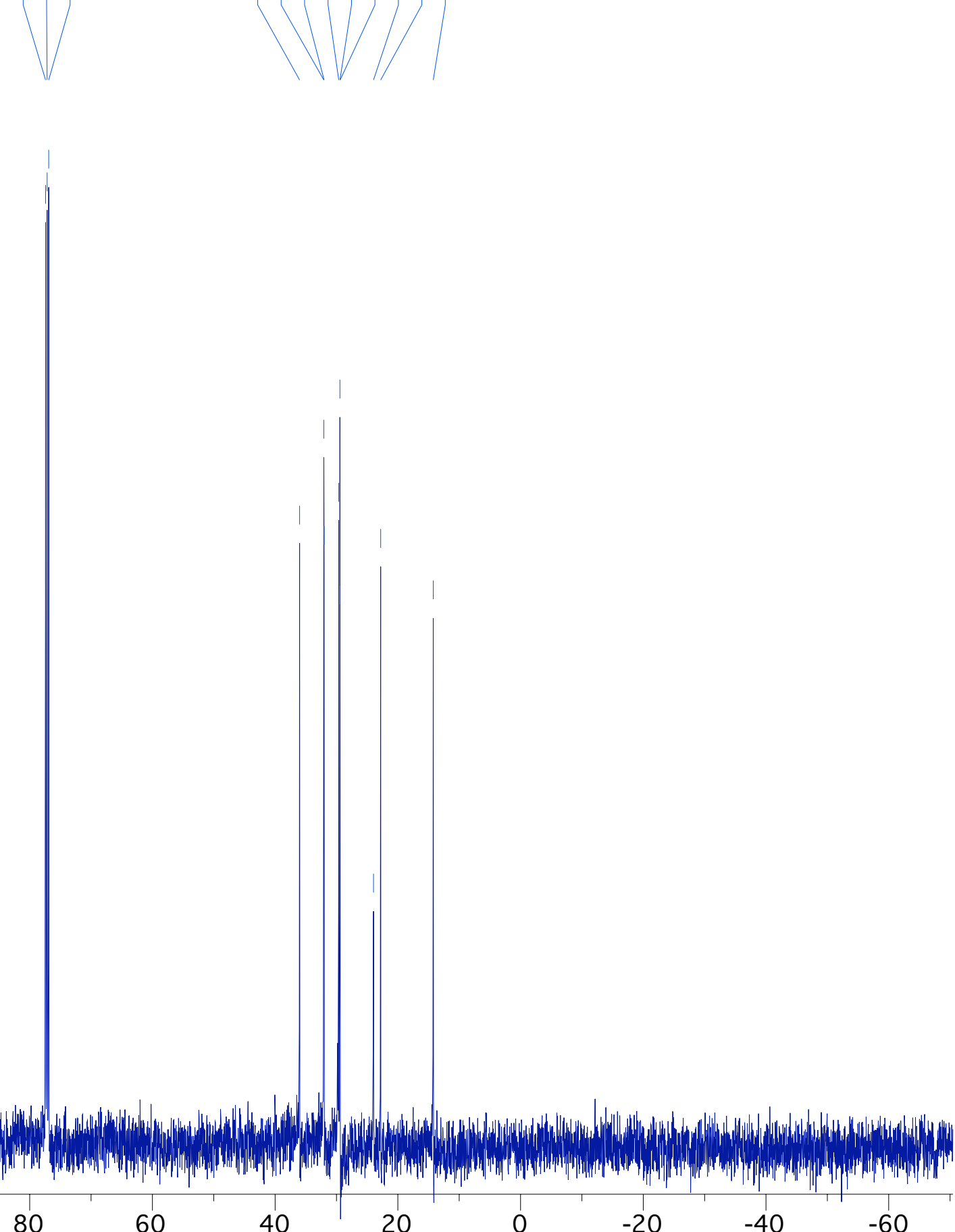

220

200

180

160

140

120

100

80

40

20

$-20$

$-40$

$-60$ 


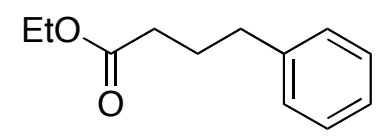

$3 j$

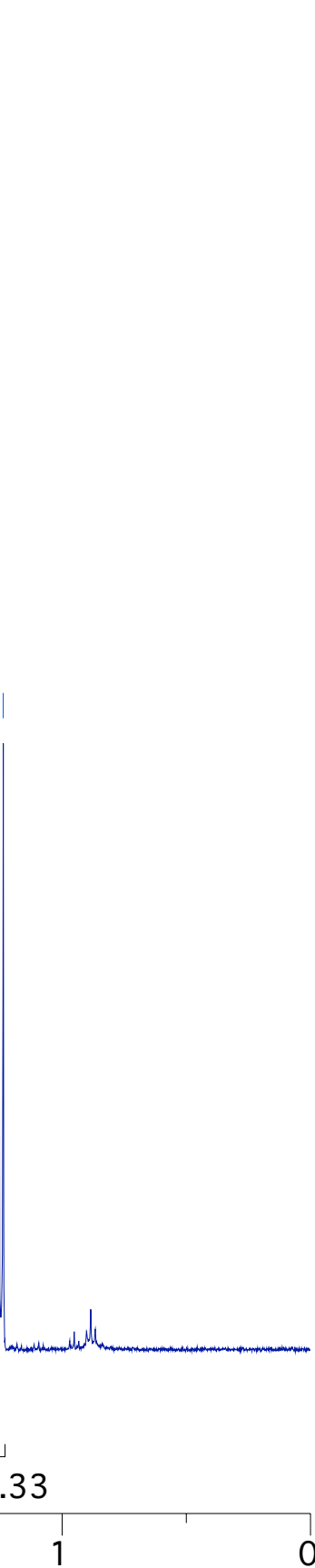

4


S38

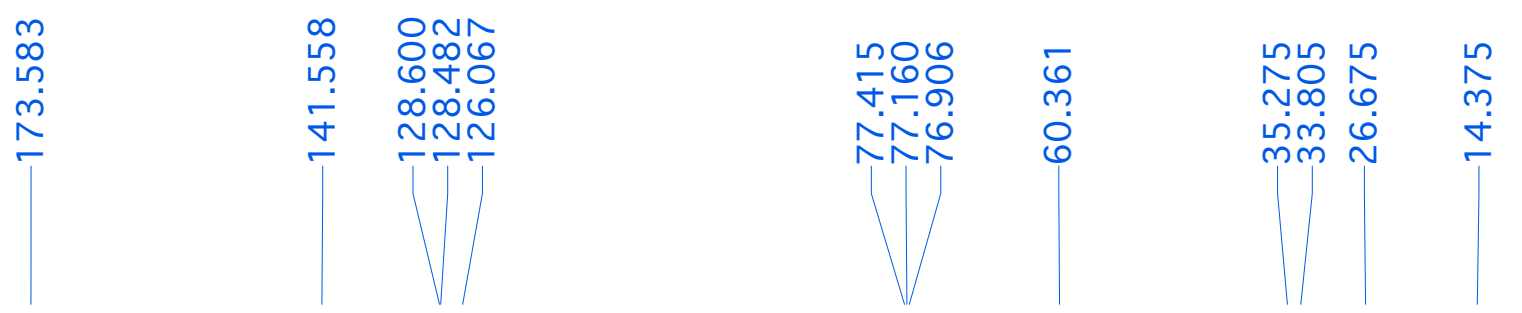<smiles>CCOC(=O)CCCc1ccccc1</smiles>

3j

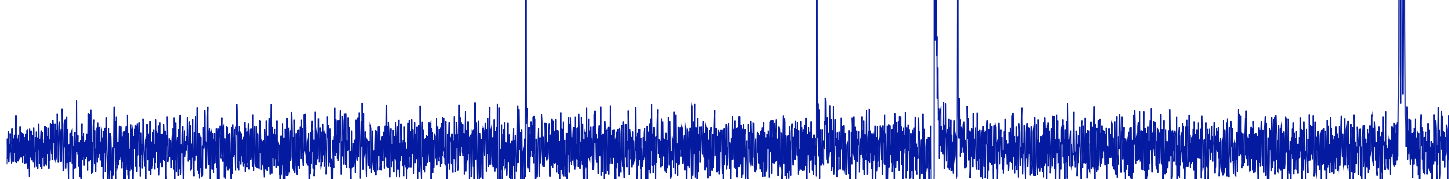



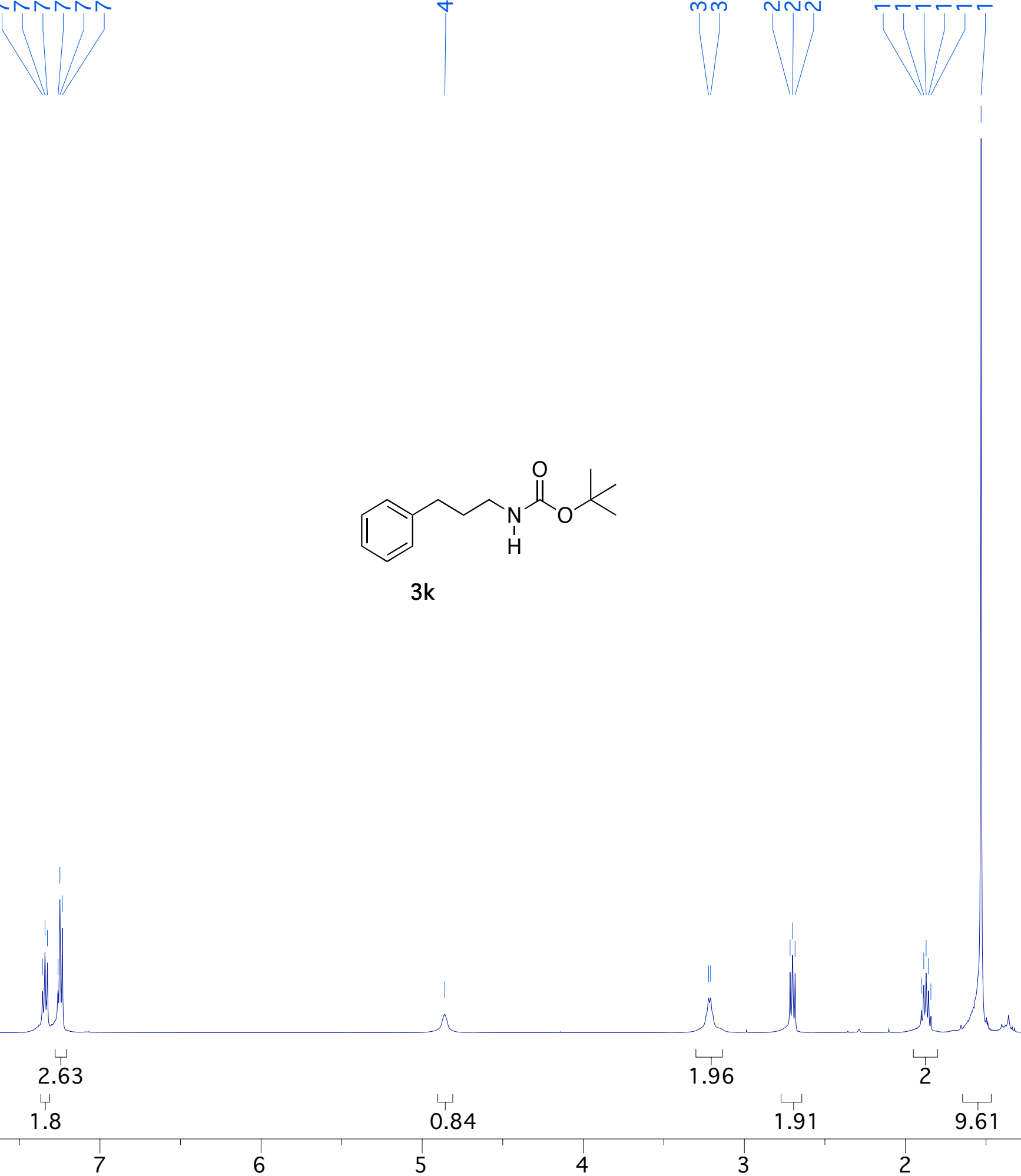


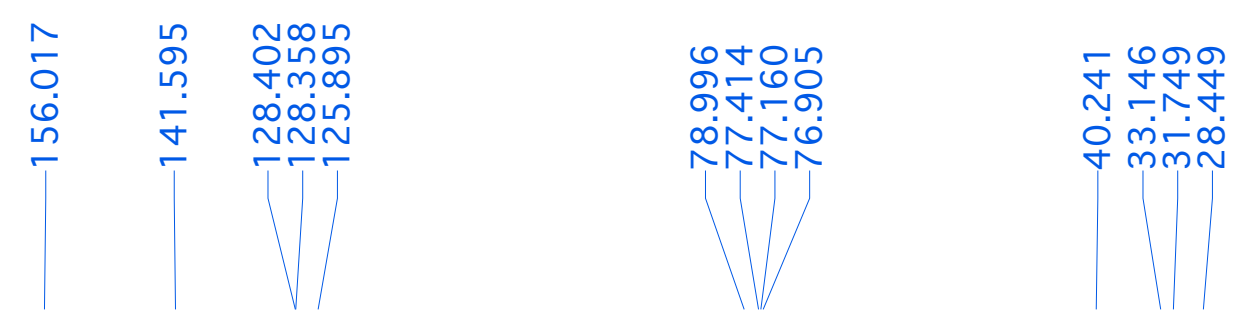

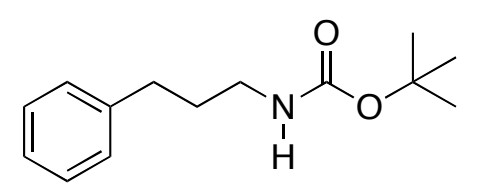

$3 k$

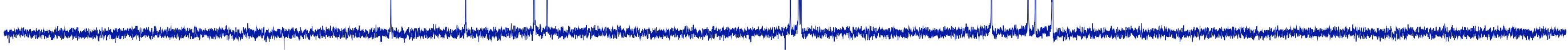




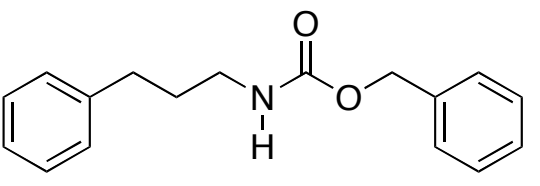

31

$$
2.87
$$$$
0.992
$$

7.26

2.35 

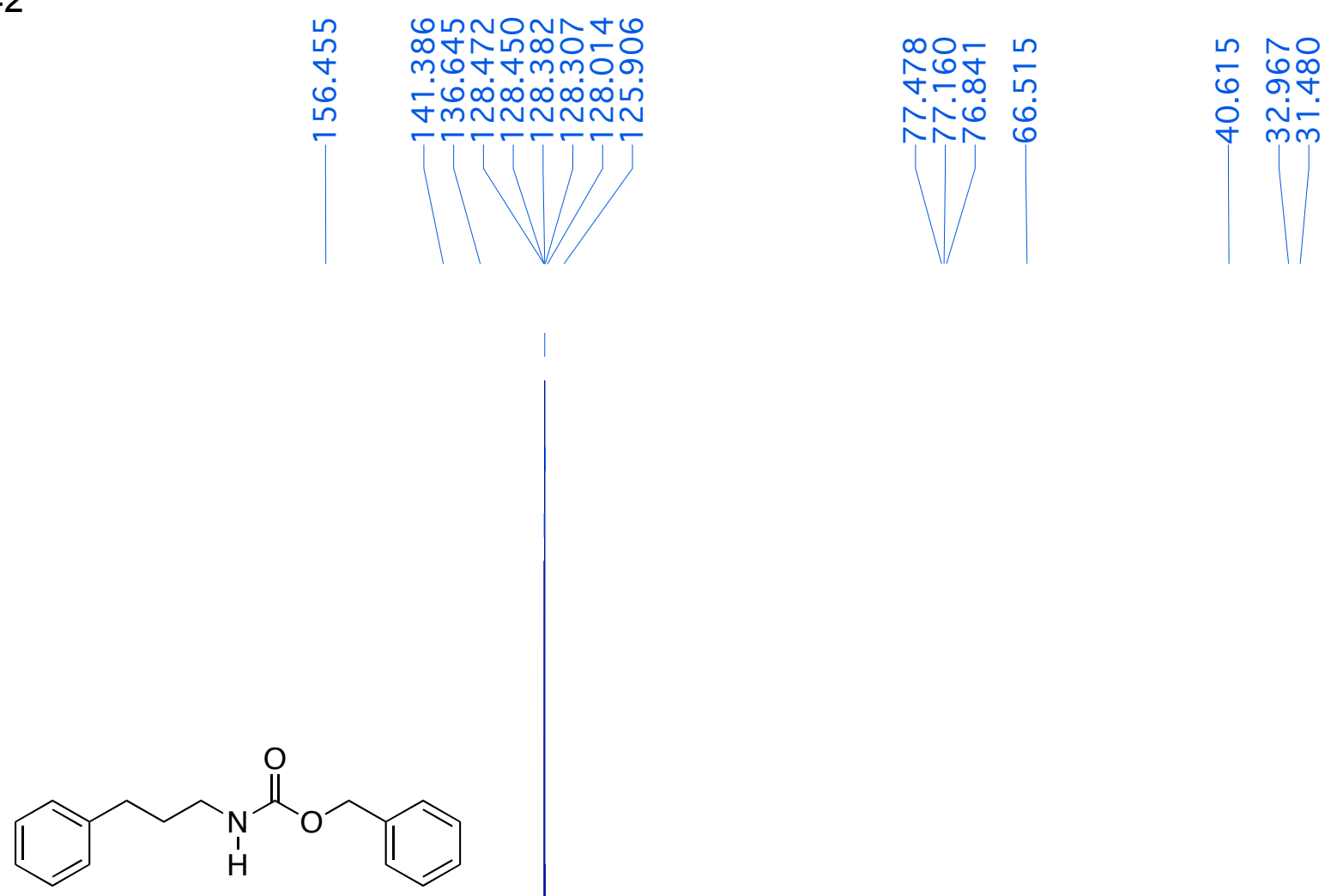

31 


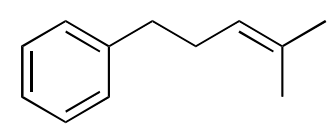

$3 m$
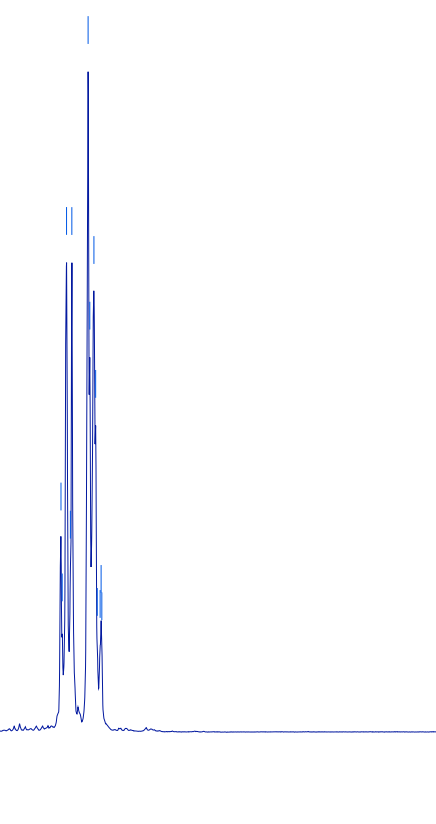

5.24 


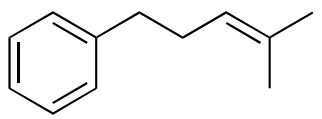

$3 m$ minn 


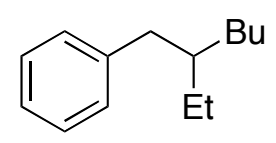

$3 n$
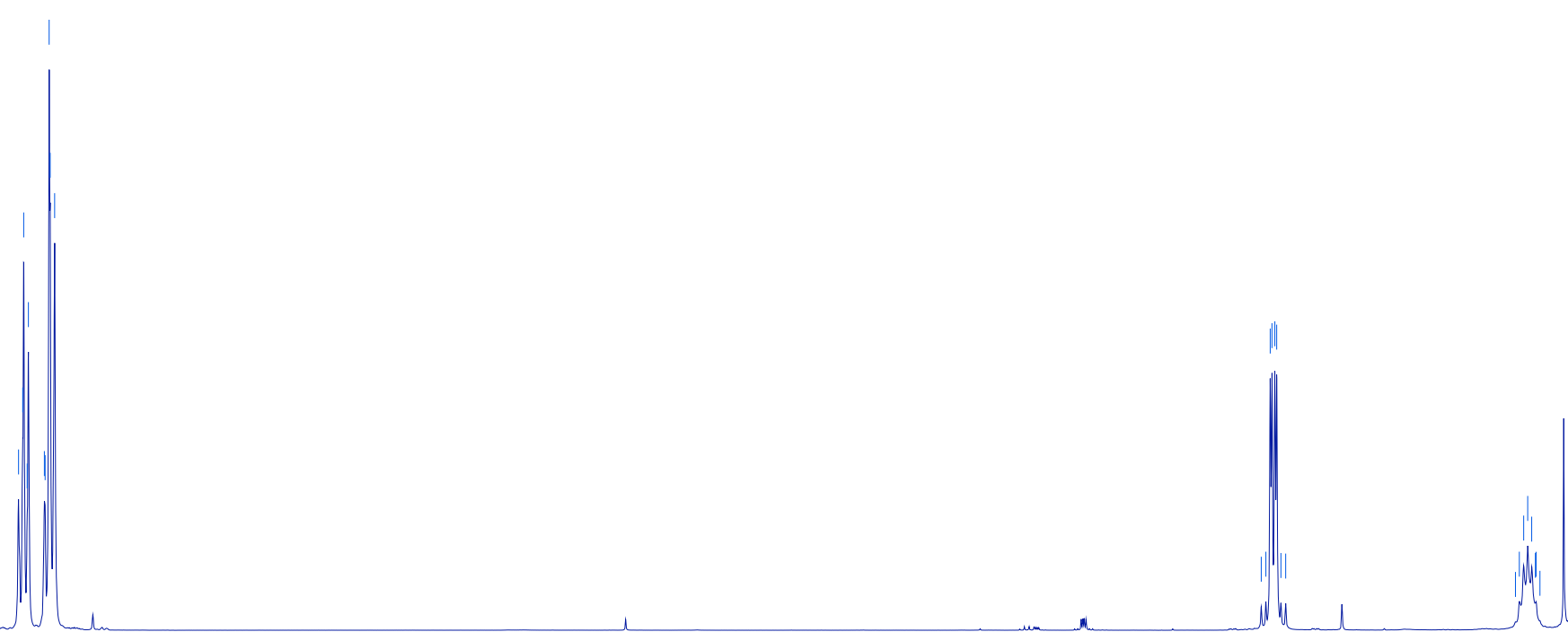

$$
2.61
$$

1.73

1.82

0.951

5.95

8

6 


\section{S46}
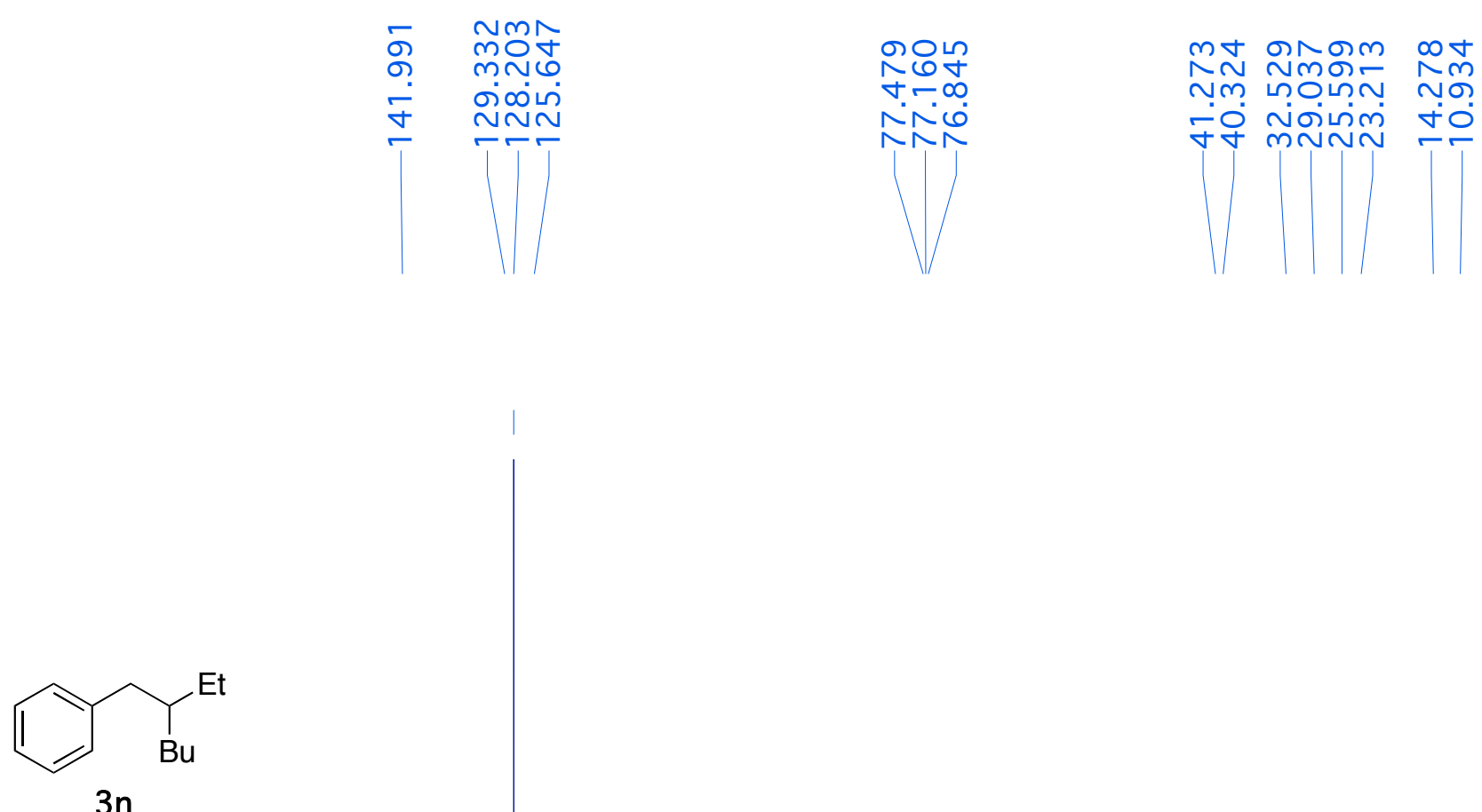

$3 n$

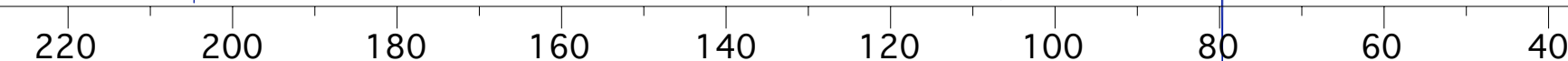

40

20

0

$-20$

$-40$

$-60$ 


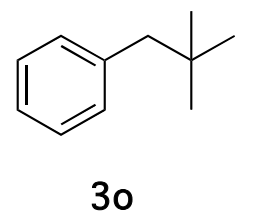




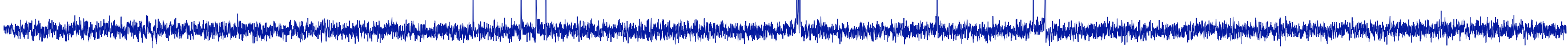




\section{S49}

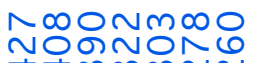

भมmmñ

NंNהNה

$3 p$

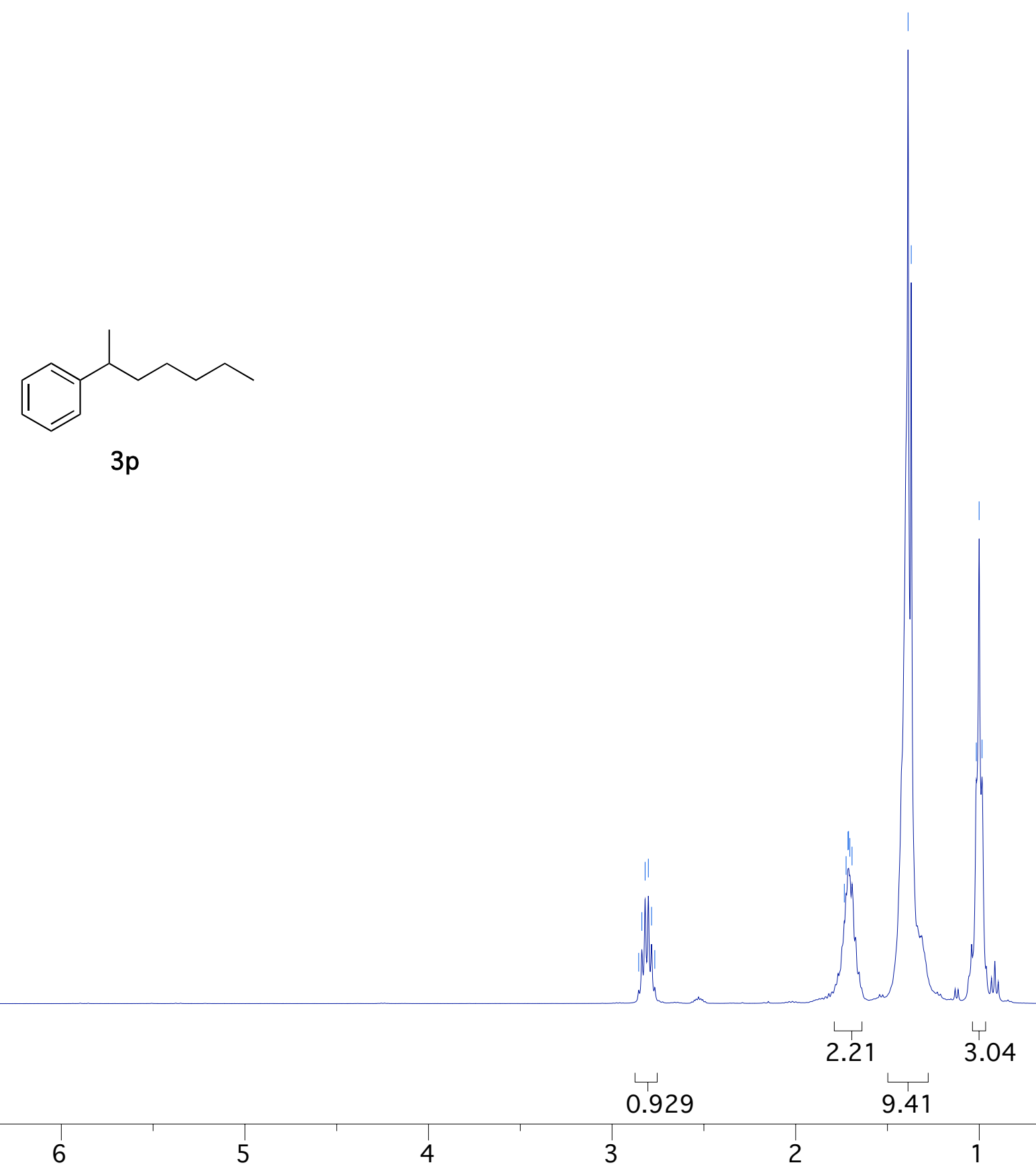

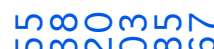

$\infty \infty \infty \infty N$

Nininin

(1) 11

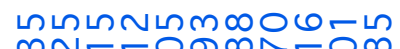

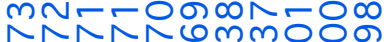

-

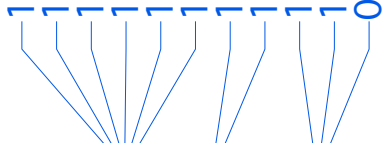



o. Ym mNNN

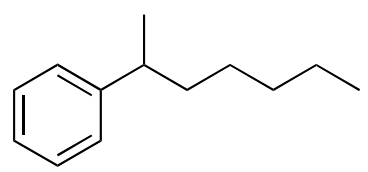

$3 p$

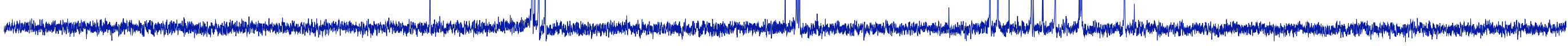

